\title{
DERIVATION OF HIGHER ORDER GRADIENT CONTINUUM THEORIES IN 2,3-D NON-LINEAR ELASTICITY FROM PERIODIC LATTICE MODELS
}

\author{
S. Bardenhagen and N. Triantafyllidis \\ Aerospace Engineering, University of Michigan, Ann Arbor, MI 48109, U.S.A.
}

(Receited 26 Fehruary 1993)

\begin{abstract}
SOLIDS THAT EXHIBIT localization of deformation (in the form of shear bands) at sufficiently high levels of strain, are frequently modeled by gradient type non-local constitutive laws, i.e. continuum theories that include higher order deformation gradients. These models incorporate a length scale for the localized deformation zone and are either postulated or justified from micromechanical considerations. Of interest here is the consistent derivation of such models from a given microstructure and the subsequent investigation of their localization and stability behavior under finite strains.

In the interest of simplicity, the microscopic model is a discrete, periodic, non-linear elastic lattice structure in two or three dimensions. The corresponding macroscopic model is a continuum constitutive law involving displacement gradients of all orders. Attention is focused on the simplest such model. namely the one whose cnergy density includes gradients of the displacements only up to the second order. The relation between the ellipticity of the resulting first (local) and second (non-local) order gradient models at finite strains, the stability of uniform strain solutions and the possibility of localized deformation zones is discussed. The investigations of the resulting continuum are done for two different microstructures, the second one of which approximates the behavior of perfect monatomic crystals in plane strain. Localized strain solutions based on the continuum approximation are possible with the first microstructure but not with the second. Implications for the stability of three-dimensional crystals using realistic interaction potentials are also discussed.
\end{abstract}

\section{INTRODUCTION}

A FEATURE SHARED BY MANY ductile solids when sufficiently strained, is the transition of their deformation field from a smoothly varying pattern into a highly localized deformation pattern in the form of a "shear band". This instability phenomenon is local, i.e. it appears at any point whose stresses reach a critical level, and it is modeled in continuum mechanics as a loss of ellipticity in the incremental equilibrium equations of the solid. The characteristic surfaces of the governing equations indicate the position of the localized deformation zones. This approach has been proposed in the context of elasticity by HADAMARD (1903) and subsequently for rate independent elastoplastic solids by Thomas (1961), Hill (1962) and Mandel (1966). Numerous works have subsequently concentrated on the study of the localization of deformation's dependence on the assumed constitutive model. For further information on this subject, the interested reader is referred to KNOWLES and STER NBERG (1977) for elastic materials and Rice (1976) for inelastic ones. 
The loss of ellipticity approach is satisfactory for predicting the critical stresses and the orientation of the deformation zone at the onset of a localized deformation. However, this methodology fails to predict the size of the localized deformation zone and it cannot provide any constitutive information about the evolution of deformation inside the localized zone. Due to the above mentioned limitations, numerical (most frequently finite element) calculations in pertaining boundary value problems show dependence of results on selected mesh size and orientation (see TVERGAARD et al., 1981 ).

To correct the above physical and numerical inadequacies of the simple loss of ellipticity approach, and given the increased importance of studying localized failure problems in mechanics, a number of remedies have been proposed. Several improvements have been suggested within the framework of classical continuum mechanics. They consist of either considering the imperfection sensitivity to pre-existing "weak zones" in the solid (see MARCINIAK and KUCZYNSKI, 1967), or incorporating viscous and thermal coupling effects in the constitutive model (see MOLINARI and CIIITON, 1987; Clifton, 1990). The alternative improvement approach involves the relaxation of the "locat action" hypothesis of classical continuum mechanics, which dictates that only the first gradient of the deformation enters the constitutive law. Continuum modcls that violate the local action hypothesis are termed "non-local" and are divided into two classes. The first class consists of integral type models whose strains and stresses at a given point depend on a convolution type integral which accounts for the history of displacements in a finite neighborhood about the point in question. The second class consists of pointwise models in which the stress at a point is calculated based exclusively on information given at this point. The simplest models in this class are higher order gradient models for which the strains and stresses at a point depend on the history of displacement gradients, up to a certain order, evaluated at the point in question.

Attention is presently focused on this last approach to modeling the localization of deformation, namely the incorporation of higher order gradients in the constitutive law. The attractions of this approach are its simplicity (no dependence on unknown weak zones in the solid or difficult to determine kernels appearing in integral type non-local models, no director fields to be postulated, no viscous or thermal effects required) and the existence of a characteristic length scale built into these models that determines the size of the localized deformation zones appearing at adequately large levels of strain. In particular, constitutive models incorporating up to the second order gradient of displacement are the simplest such models that exhibit the wanted localized strain solutions with zone widths depending on the characteristic length scale. The incorporation of a second order gradient term in the material law found many applications in the study of localization phenomena, not only for solids (see AIFANTIS, 1984 ; Coleman. 1983 ; Coleman and Hodgdon, 1985) but in fluids as well [see Van Der Waals' (1893) classical paper and also Aifantis and Serrin (1983a, b)].

The above mentioned second order gradient models, although often physically motivated, are basically phenomenological. The coefficients of the second order gradient terms are either postulated [as in TRIANTAFyllidis and AIfantis (1986)] or heuristically obtained from the assumption of continuum state equations coupling macroscopic and microscopic state variables (see MINDLIN, 1964 ; ERINGEN and SUHUBI, 
1964; Suhubi and ERINGen, 1964; Aifantis, 1987). With the recent considerable growth of higher order gradient models proposed for an ever increasing number of material behaviors, e.g. elastoplastic, viscoplastic, thermoviscoplastic, the issues of consistent derivation of the macroscopic model from the microscale one and comparison of the solutions to the same boundary value problem for the two corresponding models, become increasingly relevant.

For simplicity, attention will be restricted here only to non-linear elastic media with discrete periodic microstructures. Of interest is the consistent derivation of a macroscopic higher order gradient continuum model from the properties of the corresponding lattice microstructure. Although derivations of higher order gradient continuum theories based on discrete periodic microstructures are not novel, especially in the Physics literature (see Askar, 1985; Kunin, 1982; Minduin, 1965; TouPIN and GAZIS, 1965), attention has been focused on linear theories, static or dynamic. First order gradient (local) continuum calculations for non-linear periodic lattice structures are also frequently encountered in the Physics and Material Science literature, in particular when elastic properties of crystals based on atomistic pair potentials are sought (see BORN and HUANG, 1954; WeINER, 1983; MiLSTEIN and HiLl, 1977, 1978 , 1979). The derivation of higher order gradient continuum theories from the non-linear periodic microstructures of interest here does not seem to have attracted attention so far, to the best of our knowledge. The same comment applies to the comparison between the continuum and discrete solutions to boundary value problems for the above discussed microstructures.

As a first step in this direction, TriantafyLlidis and BardenHagen (1993) have studied a discrete one-dimensional non-linear elastic periodic microscopic model and consistently derived the corresponding continuum higher order gradient models. The resulting simplest possible such model that takes the microstructural scale into account (i.e. the lattice size) is the second order gradient one. This continuum model which was derived from a given microstructure has been found to give results in excellent agreement with the corresponding exact microscopic model for localized deformation solutions until the localization zone begins to propagate through the structure. The present work is a generalization of the above mentioned one-dimensional class of models to two, and three-dimensional lattice structures.

The contents of this paper are as follows: Section 2 details the general periodic elastic lattice model and outlines the derivation of the continuum macroscopic model from the properties of the discrete microscopic model. The Euler--Lagrange equations and boundary conditions for boundary value problems involving these continuum models are derived for the two simplest such models, namely the ones that contain the lowest order term in the lattice size. Both continuum models are equivalent in the sense that they differ by a null Lagrangian and hence they produce the same EulerLagrange equations of equilibrium. The simplest of these two equivalent formulations contains deformation gradients only up to the second order and is used in all the subsequent investigations reported here. The relations between the ellipticity condition for the first (local) and second (non-local) order gradient continuum models and their implication on stability are also discussed. A localized deformation is possible when the non-local continuum model remains elliptic at strains corresponding to the loss of ellipticity of the local model, here termed for convenience as "critical strains". 
When the non-local model is not elliptic at critical strains, we show that the unilorm critical strain solution under displacement control is no longer stable and hence the local continuum approximation is no longer valid near critical strain levels.

Section 3 presents two particular lattice examples, derives the corresponding continuum second order gradient models and investigates the ellipticity of the second order gradient models. For the first example the non-local (second order gradient) model is elliptic at critical strains, thus implying the possibility of a localized deformation zone. For the second example, a two-dimensional model of a perfect monatomic crystal in plane strain, the corresponding non-local continuum is not elliptic at critical strains. As a result we conclude the impossibility of reaching a uniform critical strain in that model due to the instabilities that should appear prior to reaching the critical strain level in a displacement controlled experiment. The local continuum approximation is therefore invalid for strain levels in the neighborhood of the critical ones and hence there is no need to investigate the ellipticity of the continuum model based on any higher order gradient term. Section 4 concludes the presentation with a discussion of the results and comments about the necessary modifications for calculations modeling perfect monatomic crystals in three dimensions.

\section{Derivation of Higher Order Gradient Model in 3-D}

The derivation of the continuum macroscopic model from the corresponding discrete microscopic model is the same in two and three dimensions. Here the derivation is presented in three dimensions, but the results for the two-dimensional case are easily recovered from their three-dimensional counterparts when the range of the script Latin indices $i, j, k, \ldots$ is from 1 to 2 (instead of $1-3$ ). The Einstein summation convention over repeated script Latin indices is adopted in the rest of this work. A repeated lower case Latin index implies summation from 1 to 3 unless explicitly stated otherwise. Summation over upper case Latin indices, which correspond to nodes, will be indicated explicitly.

\subsection{Discrete model description}

Consider an infinite three-dimensional, regular, orthogonal grid aligned with a Cartesian coordinate system. The grid spacings along the $X_{i}$-directions are denoted by $r_{i} \varepsilon$ where $\varepsilon$ is the diagonal dimension of the unit cell and $r_{i} r_{i}=1$. The discrete micromechanical model is a periodic structure composed of interacting nodes which, in the undeformed configuration, are located at (not necessarily all) grid points, i.e. the nodes form a lattice structure. Grid points are referenced by the integer triplets $I=\left(i_{1}, i_{2}, i_{3}\right)$ corresponding to grid point positions $\left(i_{1} r_{1} \varepsilon_{1} i_{2} r_{2} \varepsilon_{1} i_{3} r_{3} \varepsilon\right)$. The reference position vector (in the undeformed configuration) for node $I$ is denoted by $\mathbf{X}_{I}$ and has components $X_{l j}=i_{i} r_{j} a$ (no sum). The current position vector (in the deformed

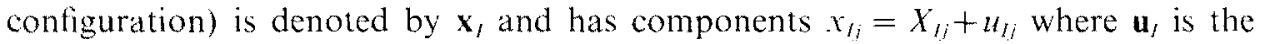
displacement vector for node 1 .

More useful for the description of the above introduced lattice are relative position vectors. For nodes $I$ and $J$, the reference relative position vector of node $J$ with respect 
to node $I$ is $\Delta \mathbf{X}_{N}=\mathbf{X}_{J}-\mathbf{X}_{I}$ and has components $\Delta X_{N k}=X_{J k}-X_{J k}=\varepsilon \Delta_{N k}$ where the relative index $N$ is defined by $N=J-I\left(n_{l}=j_{l}-i_{l}\right)$ and $\boldsymbol{\Delta}_{N}=\Delta \mathbf{X}_{N} / \varepsilon$ is the dimensionless reference relative position vector of node $J$ with respect to node $I$ with components $\Delta_{N i}=r_{i} n_{i}$ (no sum). The distance between nodes $J$ and $I$ in the reference configuration, $L_{N}$, is given by:

$$
L_{N}=\left\|\Delta \mathbf{X}_{N}\right\|=\left(\Delta X_{N k} \Delta X_{N k}\right)^{1 / 2}=\varepsilon\left(\Delta_{N k} \Delta_{N k}\right)^{1 / 2},
$$

where \|\| indicates the norm of vector $\mathbf{v}\left(\|\mathbf{v}\|=(\mathbf{v} \cdot \mathbf{v})^{1: 2}=\left(v_{i} v_{i}\right)^{1 / 2}\right)$. The current relative position vector of node $J$ relative to node $I$ is $\Delta \mathbf{x}_{N}=\mathbf{x}_{J}-\mathbf{x}_{I}=\varepsilon \Delta_{N}+\mathbf{u}_{J}-\mathbf{u}_{I}$ and has components $\Delta x_{N k}=x_{J k}-x_{l k}=\varepsilon \Delta_{N k}+u_{J k}-u_{l k}$. The current distance between nodes $J$ and $I, l_{N}$, is given by:

$$
l_{N}=\left\|\Delta \mathbf{x}_{N}\right\|=\left(\Delta x_{N k} \Delta x_{N k}\right)^{1 / 2} \text {. }
$$

Each node $I$ experiences a central force (along the current position vector $\Delta \mathbf{x}_{N}$ ) due to neighbor $J$. The interaction force is derivable from the interaction potential $\phi_{N}\left(l_{N}\right)$. The lattices under consideration are taken to be point symmetric, i.e. for each node $I$, if node $J=I+N$ is in the lattice, also node $I-N=2 I-J$ is in the lattice, and $\phi_{N}(\cdot)=\phi_{-N}(\cdot)$ (see Fig. 1 for an illustration in two dimensions). Interaction potentials are assumed to have a finite range, so that each node is influenced only by a finite number of neighbors.

A convenient strain parameter $\beta_{N}$ (and not necessarily the only such choice) which measures strain between nodes $J$ and $I$, is given by:

$$
\beta_{N}=\frac{1}{2}\left[\left(l_{N} / L_{N}\right)^{2}-1\right]
$$

Interaction potentials $\phi_{N}\left(l_{N}\right)$ may thus be written in terms of dimensionless potentials $w_{N}\left(\beta_{N}\right)$ multiplied by the unit cell volume $r_{1} r_{2} r_{3} \varepsilon^{3}$ and a common modulus $E$ :

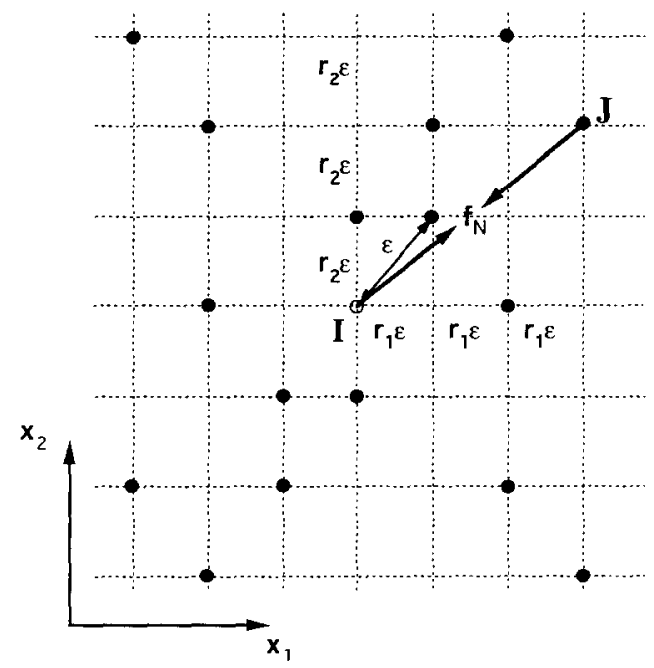

Fig. 1. Illustration in two dimensions of a unit cell which is point symmetric about its center node, here depicted in white. The nodes which interact with the center node are depicted in black. The grid spacings are $r_{1} \varepsilon$ in the $X_{1}$ direction and $r_{2} \varepsilon$ in the $X_{2}$ direction. 


$$
\phi_{N}\left(l_{N}\right)=r_{1} r_{2} r_{3} \varepsilon^{3} E w_{N}\left(\beta_{N}\right) .
$$

The dimensionless interaction potential function $w_{N}\left(\beta_{N}\right)$ may be calculated from any interaction potential. Two such potential functions are of particular interest in this investigation and will be examined in more detail in Section 3.

The equilibrium cquations for node $I$, tcrmed the "center node" and depicted in white in Fig. 1, are given in the absence of external forces by:

$$
\sum_{J} \mathbf{f}_{I \ldots I}=\sum_{N} \mathbf{f}_{N}=\mathbf{0}
$$

where the summations are over all nodes $J$, termed "interacting nodes" and depicted in black in Fig. I, interacting with node $I$. The central force experienced by node $I$ due to the influence of node $J$ is given in dyadic and component form by:

$$
\begin{aligned}
\mathbf{f}_{N} & =\frac{\partial \phi_{N}}{\partial \mathbf{u}_{N}}=r_{1} r_{2} r_{3} \varepsilon^{3} E \frac{\Delta \mathbf{x}_{N}}{L_{N}^{2}} w_{N}^{\prime}\left(\beta_{N}\right), \\
f_{N k} & =r_{1} r_{2} r_{3} \varepsilon^{3} E \frac{\Delta x_{N k}}{L_{N}^{2}} w_{N}^{\prime}\left(\beta_{N}\right),
\end{aligned}
$$

where the prime $(\cdot)^{\prime}$ denotes differentiation with respect to a function's argument.

\subsection{Continuum approximation of the energy density}

Numerical solution of the discrete equilibrium equations (2.5) quickly becomes expensive and lengthy for solids with dimensions much larger than that of the unit cell (solid's volume $V \gg r_{1} r_{2} r_{3} \varepsilon^{3}$ ), which is the case of interest. Rather than solve the discrete equilibrium equations for nodal displacements $\mathbf{u}_{l}$, an equivalent continuum problem for displacement $\mathbf{u}(\mathbf{X})$ is sought whose solution coincides with the solution to $(2.5)$ at the nodal points, i.e. $\mathbf{u}\left(\mathbf{X}_{I}\right)=\mathbf{u}_{I}$. When the node spacings become small, i.e. $\varepsilon \rightarrow 0$, the discrete system of algebraic equations (2.5) can be approximated by a system of differential equations for $\mathbf{u}(\mathbf{X})$ [e.g. see ASKAR (1985), Kunin (1982) for the linear case]. Of particular interest here is the extension of the one-dimensional results in TRIANTAFYLLIDIS and BARDENHAGiFN (1993) to three dimensions, i.e. the derivation of the simplest higher order gradient continuum model whose energy density, within an accuracy of $O\left(\varepsilon^{4}\right)$, is :

$$
\begin{aligned}
\widehat{W} & =W(\mathbf{F})+\frac{\varepsilon^{2}}{2} \mathbf{G}: \mathbf{B}(\mathbf{F}): \mathbf{G} \\
& =W(\mathbf{F})+\frac{\varepsilon^{2}}{2} B_{i j k i m n}(\mathbf{F}) u_{i, j k} u_{l . m n}
\end{aligned}
$$

where $\mathbf{F}$ is the deformation gradient of the continuum model with components $F_{i j}=\delta_{i j}+u_{i, j}$, and $\mathbf{G} \equiv \mathbf{F} \nabla$ with components $G_{i j k}=u_{i, j k}$. Subscripts following commas denote partial differentiation with respect to Cartesian coordinates $X_{i}$. Moreover Cartesian tensors are used throughout this work for which the double dot product is defined as $\mathbf{A}: \mathbf{D}=A_{i \ldots j p q} D_{p q k \ldots l} \mathbf{e}_{i} \ldots \mathbf{e}_{j} \mathbf{e}_{k} \ldots \mathbf{e}_{i}$, the triple dot product as $\mathbf{A}: \mathbf{D}=A_{i \ldots i r q r} D_{p q r k \ldots l} \mathbf{c}_{i} \ldots \mathbf{c}_{j} \mathbf{c}_{k} \ldots \mathbf{c}_{l}$, etc. 
To find the relations between $W(\mathbf{F}), \mathbf{B}(\mathbf{F})$ of the continuum model and the properties of the discrete model [i.e. the dimensionless interaction potentials $w_{N}\left(\beta_{N}\right)$ ], either an equilibrium approach or an energy approach can be used as discussed in TRIANTAFYllidis and Bardenhagen (1993). Here the energy approach is employed. A continuum energy density of the form (2.7) can be derived directly from the interaction potentials of all lattice nodes interacting with node $I$.

The ensuing derivation provides a continuum energy density up to any order of $\varepsilon$ desired. Of interest here is the lowest order correction, i.e. the $O\left(\varepsilon^{2}\right)$ term, for it introduces the scale of the microstructure $\varepsilon$ in the simplest way.

The energy density of the discrete structure, denoted by $\bar{W}$, equals half the sum of the energies of all nodes interacting with node $I$ (the other half corresponding to nodes $J=I+N$ ), divided by the unit cell volume :

$$
\bar{W}=\frac{1}{2} \sum_{N} \frac{\phi_{N}\left(l_{N}\right)}{r_{1} r_{2} r_{3} \varepsilon^{3}}=\frac{E}{2} \sum_{N} w_{N}\left(\beta_{N}\right) .
$$

An adequately smooth continuous function $\mathbf{u}(\mathbf{X})$ is assumed that approximates equilibrium displacements $\mathbf{u}_{\text {, }}$ at the nodal points $\mathbf{X}_{J}$, i.e. $\mathbf{u}\left(\mathbf{X}_{J}\right) \cong \mathbf{u}_{J}$. Using the Taylor series expansion for $\mathbf{x}=\mathbf{X}+\mathbf{u}$, about $\mathbf{X}_{l}$, to evaluate the current relative position vectors $\Delta \mathbf{x}_{N}=\varepsilon \boldsymbol{\Delta}_{N}+\mathbf{u}_{J}-\mathbf{u}_{l}$, one obtains :

$$
\begin{aligned}
& \Delta \mathbf{x}_{N}=\varepsilon \mathbf{F} \cdot \boldsymbol{\Delta}_{N}+\frac{\varepsilon^{2}}{2} \mathbf{G}: \Delta_{N} \boldsymbol{\Delta}_{N}+\frac{\varepsilon^{3}}{6} \mathbf{H}: \boldsymbol{\Delta}_{N} \boldsymbol{\Delta}_{N} \boldsymbol{\Delta}_{N}+\cdots, \\
& \Delta x_{N i}=\varepsilon F_{i j} \Delta_{N j}+\frac{\varepsilon^{2}}{2} u_{i, j k} \Delta_{N j} \Delta_{N k}+\frac{\varepsilon^{3}}{6} u_{i, j k i} \Delta_{N j} \Delta_{N k} \Delta_{N l}+\cdots,
\end{aligned}
$$

where all derivatives are evaluated at $\mathbf{X}_{I}$ and where $\mathbf{G} \equiv \mathbf{F} \nabla, \mathbf{H} \equiv(\mathbf{F V}) \nabla$ with components $G_{i j k}=u_{i, j k}$ and $H_{i j k l}=u_{i, j k l}$ respectively.

Substitution of (2.1)-(2.3) and (2.9) into (2.8) and subsequent expansion of the result in terms of ascending powers of $\varepsilon$ yields :

$$
\begin{aligned}
W & =W(\mathbf{F})-\frac{\varepsilon^{2}}{2}[3 \mathbf{G}: \mathbf{B}(\mathbf{F}): \mathbf{G}+\mathbf{C}(\mathbf{F}) ! \mathbf{H}]+O\left(\varepsilon^{4}\right) \\
& =W(\mathbf{F})-\frac{\varepsilon^{2}}{2}\left[3 B_{i j k l m n}(\mathbf{F}) u_{i, j k} u_{l, m n}+C_{i j k l}(\mathbf{F}) u_{i, j k l}\right]+O\left(\varepsilon^{4}\right) .
\end{aligned}
$$

where

$$
\begin{gathered}
W(\mathbf{F})=\frac{E}{2} \sum_{N} w_{N}\left(\bar{\beta}_{N}\right), \quad \bar{\beta}_{N}(\mathbf{F})=\frac{1}{2}\left[\frac{F_{i j} \Delta_{N j} F_{i k} \Delta_{N k}}{\Delta_{N s} \Delta_{N s}}-1\right] \\
B_{i j k i m n}(\mathbf{F})=-\frac{E}{2} \sum_{N} \frac{1}{12}\left[\frac{w_{N}^{\prime \prime}\left(\bar{\beta}_{N}\right)}{\left(\Lambda_{N s} \Lambda_{N s}\right)^{2}} F_{i p} \Delta_{N p} F_{l i} \Delta_{N q}+\delta_{i l} \frac{w_{N}^{\prime}\left(\bar{\beta}_{N}\right)}{\Delta_{N s} \Lambda_{N s}}\right] \Delta_{N j} \Delta_{N k} \Delta_{N m} \Delta_{N n} \\
C_{i j k i}(\mathbf{F})=-\frac{E}{2} \sum_{N} \frac{1}{3} \frac{w_{N}^{\prime}\left(\bar{\beta}_{N}\right)}{\left(\Delta_{N s} \Delta_{N s}\right)} F_{i p} \Delta_{N p} \Delta_{N j} \Delta_{N k} \Delta_{N l} .
\end{gathered}
$$


The absence of an $O(\varepsilon)$ term in $W$ (and, more generally, of all odd order terms in $\varepsilon$ ) is due to the point symmetry requirements specified in Section 2.1.

Notice that the energy density $W$ as given by $(2.10),(2.11)$ is not of the form (2.7). However, as suggested by the energy density forms encountered in the onedimensional case (see Triantafyllidis and BARDENHAGEN, 1993) one observes that in the three-dimensional case the $O\left(\varepsilon^{2}\right)$ term of $\tilde{W}-W$ :

$$
4 \mathbf{G}: \mathbf{B}(\mathbf{F}): \mathbf{G}+\mathbf{C}(\mathbf{F}) ! \mathbf{H}=4 B_{i j k t_{m} m}(\mathbf{F}) u_{i, j k} u_{l, m n}+C_{i j k l}(\mathbf{F}) u_{i, j k l}
$$

is also a null Lagrangian, i.c. its corresponding Euler-Lagrange equations are identically zero. This may be verified directly by noting the symmetries of $B_{i j k / m, k}$ and $C_{i / k}$ given in (2.11), specifically that:

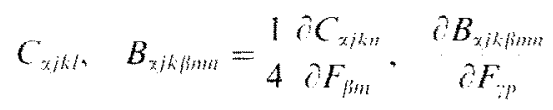

are symmetric with respect to any interchange of Latin or Greek indices. Thus the simplest form for the continuum energy density corresponding to the microscopic model at hand is the one given by $(2.7)$ with an accuracy of $O\left(i^{4}\right)$.

The continuum equilibrium equations corresponding to energy density forms $\widehat{W}(\mathbf{F}, \mathbf{G})$ or $W(\mathbf{F}, \mathbf{G}, \mathbf{H})$ will be derived in Section 2.3. It should be noted that both energy densities are frame invariant, as is easily verified $\left(\widehat{W}(\mathbf{F}, \mathbf{F} V)=\widehat{W}\left(\mathbf{F}^{*}, \mathbf{F}^{*} \nabla\right)\right.$, $W(\mathbf{F}, \mathbf{F} \nabla,(\mathbf{F} \nabla) \nabla)=W\left(\mathbf{F}^{*}, \mathbf{F}^{*} \nabla,\left(\mathbf{F}^{*} \nabla\right) \nabla\right)$, for $\mathbf{F}^{*}=\mathbf{Q} \cdot \mathbf{F}$ where $\mathbf{Q}$ is any independent of $X$ orthogonal tensor). It should also be noted here that the same equilibrium equations, which are presented in the next section, can be obtained directly from the discrete equilibrium equations (2.5), (2.6) by substituting (2.1) (2.3) and (2.9), subsequently expanding the result in terms of ascending powers of $a$ and then using symmetry relations $(2.12)$, a rather formidable computation.

\subsection{Cominum model boundary value problem}

In this section the boundary value problems corresponding to energy densities $\widehat{W}$ and $W$ will be derived. While both energy densities yield the same equilibrium equations, the boundary conditions are in general different. Energy density $\widehat{W}$ is preferred over $W$ in view of its simpler form and its frequent use in previous investigations.

Noticing that $\widehat{W}$ and $W$ can be put in the general form $W(\mathbf{F})+\left(s^{2} / 2\right) h(\mathbf{F}, \mathbf{G}, \mathbf{H})$, the differences in boundary conditions are presented most clearly by using the general higher order gradient term $\left(\varepsilon^{2} / 2\right) h(\mathbf{F}, \mathbf{G}, \mathbf{H})$ in the boundary value problem derivations. The boundary conditions pertaining to energy densities $\widehat{W}$ and $W$ are obtained by selecting the appropriate expressions for $h(\mathbf{F}, \mathbf{G}, \mathbf{H})$ from (2.7) or (2.10).

The potential energy of a body which in the reference configuration occupies volume $V$, in the absence of body forces and boundary loads (for a displacement controlled problem), is then

$$
\theta=\int_{r}\left[W(\mathbf{F})+\varepsilon_{2}^{2} h(\mathbf{F}, \mathbf{G}, \mathbf{H})\right] \mathrm{d} V
$$


The equilibrium equations and boundary conditions are found by extremizing $\mathscr{E}$ over all admissible displacements $\mathbf{u}(\mathbf{X})$. A standard calculation from $\mathscr{E}_{\mathbf{a}} \cdot \delta \mathbf{u}=\mathbf{0}$ gives

$$
\begin{aligned}
& -\int_{V} \delta \mathbf{u} \cdot\left\{\left(\frac{\partial W}{\partial \mathbf{F}}+\frac{\varepsilon^{2}}{2}\left[\frac{\partial h}{\partial \mathbf{F}}-\frac{\partial h}{\partial \mathbf{G}} \cdot \nabla+\left(\frac{\partial h}{\partial \mathbf{H}} \cdot \nabla\right) \cdot \nabla\right]\right) \cdot \nabla\right\} \mathrm{d} V \\
& +\int_{h V} \delta \mathbf{u} \cdot\left\{\frac{\partial W}{\partial \mathbf{F}}+\frac{\varepsilon^{2}}{2}\left[\frac{\partial h}{\partial \mathbf{F}}-\frac{\partial h}{\partial \mathbf{G}} \cdot \nabla+\left(\frac{\partial h}{\partial \mathbf{H}} \cdot \nabla\right) \cdot \nabla\right]\right\} \cdot \mathbf{n} \mathrm{d} S \\
& +\int_{h V}(\delta \mathbf{u} \nabla):\left\{\frac{\varepsilon^{2}}{2}\left[\frac{\partial h}{\partial \mathbf{G}}-\frac{\partial h}{\partial \mathbf{H}} \cdot \nabla\right]\right\} \cdot \mathbf{n} \mathrm{d} S+\int_{\partial V}((\delta \mathbf{u} \nabla) \nabla): \frac{\varepsilon^{2}}{2} \frac{\partial h}{\partial \mathbf{H}} \cdot \mathbf{n} \mathrm{d} S=\mathbf{0}, \\
& -\int_{V} \delta u_{i}\left\{\frac{\partial}{\partial X_{i}}\left(\frac{\partial W}{\partial F_{i j}}+\frac{\varepsilon^{2}}{2}\left[\frac{\partial h}{\partial F_{i j}}-\frac{\partial}{\partial X_{k}}\left(\frac{\partial h}{\partial G_{i j k}}\right)+\frac{\partial^{2}}{\partial X_{k} \partial X_{l}}\left(\frac{\partial h}{\partial H_{i j k l}}\right)\right]\right)\right\} \mathrm{d} V
\end{aligned}
$$

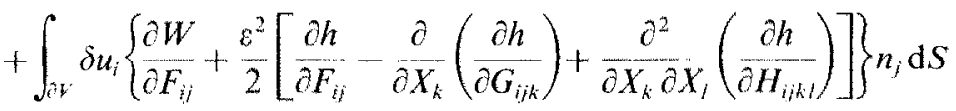

$$
\begin{aligned}
& +\int_{h y} \delta u_{i, j}\left\{\frac{\varepsilon^{2}}{2}\left[\frac{\partial h}{\partial G_{i j k}}-\frac{\partial}{\partial X_{i}}\left(\frac{\partial h}{\partial H_{i j k}}\right)\right]\right\} n_{k} \mathrm{~d} S+\int_{i y} \delta u_{i, j k}\left\{\frac{\varepsilon^{2}}{2} \frac{\partial h}{\partial H_{i j k i}}\right\} n_{f} \mathrm{~d} S=0,
\end{aligned}
$$

where $\mathbf{n}$ is the outward unit normal to the surface $\partial V$ bounding the reference volume $V$. Gauss' divergence theorem has been applied to give the surface terms in (2.13).

Noting that $\delta \mathbf{u}$ and the surface components of its gradients are not independent on the boundary, for if $\delta \mathbf{u}$ is known on the boundary $\partial V$ so are all derivatives of $\delta \mathbf{u}$ in directions tangent to the boundary, the boundary terms in (2.13) can be written in terms of only the independent quantities on $\partial V$. Proceeding as in MindurN (1965), the gradient operator may be written as the sum of a surface operator $\nabla \equiv \nabla-D \mathbf{n}$ and a normal operator $D \equiv(() \nabla) \cdot \mathbf{n}$, i.e. for any tensor $\mathbf{A}, \mathbf{A} D=(\mathbf{A} \nabla) \cdot \mathbf{n}$, so that

$$
\delta \mathbf{u} \nabla=\delta \stackrel{s}{\mathbf{u}}+(\delta \mathbf{u} D) \mathbf{n}
$$

The divergence theorem for closed surfaces is (see BRAND, 1947):

$$
\begin{aligned}
\int_{i V} \mathbf{w} \cdot \stackrel{\mathrm{s}}{\nabla} \mathrm{d} S & =\int_{V V}(\mathbf{n} \cdot \nabla)(\mathbf{w} \cdot \mathbf{n}) \mathrm{d} S, \\
\int_{i n V}\left(w_{i, i}-w_{i, j} n_{i} n_{j}\right) \mathrm{d} S & =\int_{a V} n_{i, i} w_{j} n_{j} \mathrm{~d} S
\end{aligned}
$$


which is valid for any vector $w$. Substitution of (2.14) into (2.13), after considerable manipulation and repeated application of (2.15), permits the equilibrium equations (2.16), and boundary conditions $(2.16)_{2}-(2.16)_{4}$ for the continuum to be written as

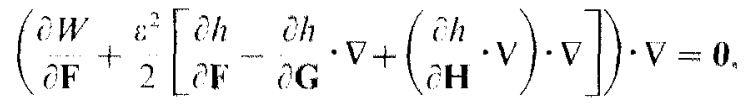

$$
\begin{aligned}
& \delta \mathbf{u} \cdot\left\{\left(\frac{\partial W}{\partial \mathbf{F}}+\frac{b^{2}}{2}\left[\frac{\partial h}{\partial \mathbf{F}}-\frac{\partial h}{\partial \mathbf{G}} \cdot \nabla+\left(\frac{\partial h}{\partial \mathbf{H}} \cdot \nabla\right) \cdot \nabla\right]\right) \cdot \mathbf{n}\right. \\
& \left.+\frac{\varepsilon^{2}}{2}\left[\left(\frac{\partial h}{\partial \mathbf{G}}-\frac{\partial h}{\partial \mathbf{H}} \cdot \nabla\right) \cdot \mathbf{n}+\left(\frac{\partial h}{\partial \mathbf{H}} \cdot \mathbf{n}\right) \cdot \mathbf{L}-\left(\frac{\partial h}{\partial \mathbf{H}} \mathbf{n n}\right) \cdot(\mathbf{n} \nabla)\right] \cdot \mathbf{L}\right\}=\mathbf{0} \\
& (\partial \mathbf{u} D) \cdot\left\{\left(\frac{\partial h}{\partial \mathbf{G}}-\frac{\partial h}{\partial \mathbf{H}} \cdot \nabla\right): \mathbf{n n}+\left(\left(\frac{\partial h}{\partial \mathbf{H}} \cdot \mathbf{n}\right) \cdot \mathbf{L}\right) \cdot \mathbf{n}+\left(\frac{\partial h}{\partial \mathbf{H}} \mathbf{n} \mathbf{n}\right) \cdot \mathbf{L}\right\}=\mathbf{0}, \\
& ((\delta \mathbf{u} D) D) \cdot\left\{\begin{array}{l}
\partial h \\
\partial \mathbf{H}
\end{array} \mathbf{n n n}\right\}=\mathbf{0},
\end{aligned}
$$

where $\mathbf{L} \equiv \mathbf{n}(\mathbf{n} \cdot \nabla)-\vec{\nabla}$, i.e. for any tensor $\mathbf{A}$, with components $A_{i} ; \dot{*}, \mathbf{A} \cdot \mathbf{L}=$

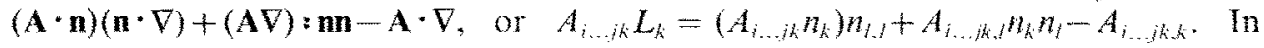
component form the above equilibrium equations and boundary conditions are:

$$
\begin{aligned}
& \frac{\partial}{\partial X_{i}}\left(\partial F_{i,}+\frac{\varepsilon^{2}}{2}\left[\frac{\partial h}{\partial F_{i j}}-\hat{\partial X_{k}}\left(\begin{array}{c}
\partial h \\
\partial G_{i k}
\end{array}\right)+\frac{\partial^{2}}{\partial X_{k} \partial X_{i}}\left(\begin{array}{c}
\partial h \\
\partial H_{i k}
\end{array}\right)\right]\right)=0, \quad i=1,2,3
\end{aligned}
$$

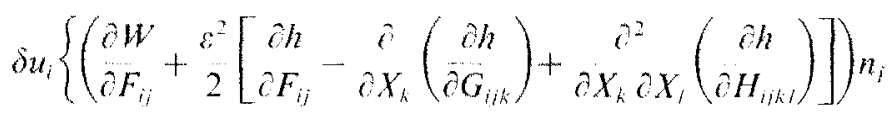

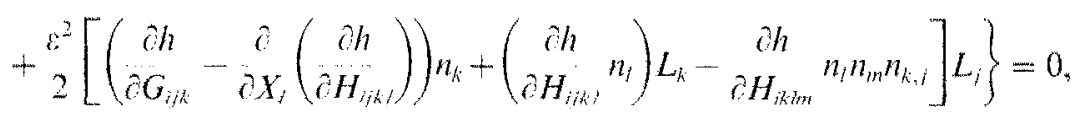

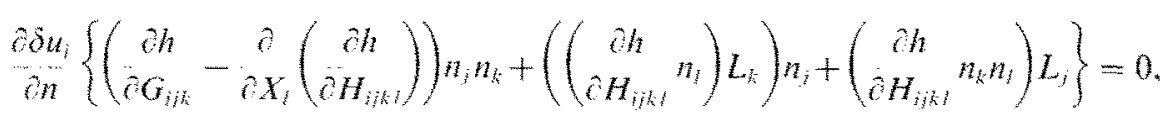

$$
\partial \partial^{2}\left\{\begin{array}{c}
\partial u_{i} \\
\partial H_{i j k}
\end{array} n_{i} n_{k} n_{i}\right\}=0
$$

where $i \delta u_{i} / \partial n=\delta u_{i .4} n_{q}$ and $\partial^{2} \delta u_{i} / \partial n^{2}=\delta u_{t .4 r} n_{q} n_{r}$ are the first and second normal derivatives of $\delta u_{i}$ respectively (directional derivatives along $\mathbf{n}$ ). Equations (2.16) take the same form (in the absence of body forces and surface loads) as those given by Mindus (1965). They are not identical equations, however, for here the energy density is a function of $\mathbf{F}, \boldsymbol{G}$ and $\boldsymbol{H}$, while in Mindlin's work (in linear elasticity) the energy density is a function of $\mathbf{e}, \mathbf{G}$ and $\mathbf{H}$, where $\mathbf{e}=(1 / 2)[\nabla \mathbf{u}+\mathbf{n} \nabla]$. 
The boundary value problem for energy density $\widehat{W}$ is found by taking [see (2.7)]:

$$
h(\mathbf{F}, \mathbf{G}, \mathbf{H})=\mathbf{G}: \mathbf{B}(\mathbf{F}): \mathbf{G} \text {. }
$$

Then $\partial h / \partial \mathbf{H}=\mathbf{0}$ and the equilibrium equations $(2.16)_{1}$ and boundary conditions $(2.16)_{2}-(2.16)_{4}$ take the form

$$
\begin{gathered}
\left(\frac{\partial W}{\partial \mathbf{F}}+\frac{\varepsilon^{2}}{2}\left[\mathbf{G}:\left(\mathbf{G}: \frac{\partial \mathbf{B}}{\partial \mathbf{F}}\right)-2(\mathbf{G}: \mathbf{B}) \cdot \nabla\right]\right) \cdot \nabla=\mathbf{0} \\
\delta \mathbf{u} \cdot\left\{\left(\frac{\partial W}{\partial \mathbf{F}}+\frac{\varepsilon^{2}}{2}\left[\mathbf{G}:\left(\mathbf{G}: \frac{\partial \mathbf{B}}{\partial \mathbf{F}}\right)-2(\mathbf{G}: \mathbf{B}) \cdot \nabla\right]\right) \cdot \mathbf{n}+\frac{\varepsilon^{2}}{2}[2 \mathbf{G}: \mathbf{B} \cdot \mathbf{n}] \cdot \mathbf{L}\right\}=\mathbf{0} \\
(\partial \mathbf{u} D) \cdot\{2 \mathbf{G}: \mathbf{B}: \mathbf{m}\}=\mathbf{0} .
\end{gathered}
$$

The boundary value problem for energy density $\bar{W}$ is found by taking [see (2.10)]:

$$
h(\mathbf{F}, \mathbf{G}, \mathbf{H})=-3 \mathbf{G}: \mathbf{B}(\mathbf{F}): \mathbf{G}-\mathbf{C}(\mathbf{F}): \mathbf{H}
$$

into equilibrium equations $(2.16)_{1}$ and boundary conditions $(2.16)_{2}-(2.16)_{4}$ which take the form

$$
\begin{aligned}
& \left(\frac{\partial W}{\partial \mathbf{F}}-\frac{\varepsilon^{2}}{2}\left[3 \mathbf{G}:\left(\mathbf{G}: \frac{\partial \mathbf{B}}{\partial \mathbf{F}}\right)+\mathbf{H}: \frac{\partial \mathbf{C}}{\partial \mathbf{F}}-6(\mathbf{G}: \mathbf{B}) \cdot \nabla+(\mathbf{C} \cdot \nabla) \cdot \nabla\right]\right) \cdot \nabla=\mathbf{0}, \\
& \delta \mathbf{u} \cdot\left\{\left(\frac{\partial W}{\partial \mathbf{F}}-\frac{\varepsilon^{2}}{2}\left[3 \mathbf{G}:\left(\mathbf{G}: \frac{\partial \mathbf{B}}{\partial \mathbf{F}}\right)+\mathbf{H}: \frac{\partial \mathbf{C}}{\partial \mathbf{F}}-6(\mathbf{G}: \mathbf{B}) \cdot \nabla+(\mathbf{C} \cdot \nabla) \cdot \nabla\right]\right) \cdot \mathbf{n}\right. \\
& \left.-\frac{\varepsilon^{2}}{2}[(6 \mathbf{G}: \mathbf{B}-\mathbf{C} \cdot \nabla) \cdot \mathbf{n}+(\mathbf{C} \cdot \mathbf{n}) \cdot \mathbf{L}-(\mathbf{C}: \mathbf{n n}) \cdot(\mathbf{n V})] \cdot \mathbf{L}\right\}=\mathbf{0} \\
& (\delta \mathbf{u} D) \cdot\{(6 \mathbf{G}: \mathbf{B}-\mathbf{C} \cdot \nabla): \mathbf{n n}+((\mathbf{C} \cdot \mathbf{n}) \cdot \mathbf{L}) \cdot \mathbf{n}+(\mathbf{C}: \mathbf{n n}) \cdot \mathbf{L}\}=\mathbf{0} \\
& ((\delta \mathbf{u} D) D) \cdot\{C: \mathbf{n n n}\}=0 \text {. }
\end{aligned}
$$

As expected from the fact that the $O\left(\varepsilon^{2}\right)$ term of the difference between energy densities $\widehat{W}$ and $\bar{W}$ is a null Lagrangian, equilbrium equations $(2.17)$, and $(2.18)$, are identical. This may be verifed directly by using the symmetry relations $(2.12)$. In component form, the equilibrim equations are laccounting for symmetry relations $(2.12)]$ :

$$
\frac{\partial}{\partial X_{s}}\left(\frac{\partial W}{\partial F_{r, s}}-\frac{g^{2}}{2}\left[\frac{\partial B_{i j k l m n}}{\partial F_{r . s}} u_{i, j k} u_{l, m n}+2 B_{i j k r s n} u_{i, j k n}\right]\right)=0, \quad r=1,2,3 .
$$

The natural boundary conditions for the two energy densities, i.e. the boundary conditions corresponding to the case where $\delta \mathbf{u}, \delta \mathbf{u} D,(\delta \mathbf{u} D) D$ are not prescribed, are generally different. One set of natural boundary conditions, the set corresponding to $(2.16)_{4}$, is satisfied identically for the boundary value problem corresponding to $\vec{W}$ (where $2 h / \partial \mathbf{H}=\mathbf{0}$ ). It is interesting to find boundary value problems, besides the 
obvious ones with full Dirichlet boundary conditions where $\delta \mathbf{u}, \delta \mathbf{u} D,(\delta \mathbf{u} D) D$ are prescribed, for which $(2.17)_{2}-(2.17)_{3}$ and $(2.18)_{2}-(2.18)_{4}$ coincide. This can be the case for certain finite lattices for which the solution to the displacement controlled problem is one of constant strain and the coordinate axes are the principal directions of deformation, or also the case for an infinite lattice for which $\mathbf{G}$ and $\mathbf{H} \rightarrow \mathbf{0}$ as $|\mathbf{X}| \rightarrow \infty$. The existence of such boundary value problems avoids the distinction between $\widehat{W}$ and $W$, and allows one to compare solutions based on a discrete model to those based on the resulting continuum model, as in TRIANTAFYLLIDIs and BARDENHAGFN (1993).

\subsection{Discussion of ellipticity for $\mathbf{W}(\mathbf{F}), \widehat{W}(\mathbf{F}, \mathbf{G})$}

As discussed in Triantafyllidis and Aifantis (1986), the essential features of the higher order gradient (also termed non-local) continuum energy density $\widehat{W}=W(\mathbf{F})+\left(\varepsilon^{2} / 2\right) \mathbf{G} \vdots \mathbf{B}(\mathbf{F}) ! \mathbf{G}$, which are responsible for the appearance of localized strain solutions, are as follows.

(i) A (local) macroscopic energy density $W(\mathbf{F})$ whose corresponding equilibrium equations, although initially strongly elliptic at $\mathbf{F}=\mathbf{I}, \uparrow$ lose ellipticity at some adequate level of strain $\mathbf{F}_{\mathrm{c}} \neq \mathbf{I}$, namely:

$$
\begin{aligned}
& (\mathbf{m n}): \begin{array}{l}
\partial^{2} W(\mathbf{I}) \\
\partial \mathbf{F} \partial \mathbf{F}
\end{array}:(\mathbf{m n})=\frac{\partial^{2} W(\mathbf{I})}{\partial F_{i l} \partial F_{k,}} m_{i} n_{j} m_{k} n_{l}>0 . \quad \forall \mathbf{m}, \mathbf{n} \in \mathbb{B}^{3} ; \quad\|\mathbf{m}\|=\|\mathbf{n}\|=1, \\
& \left(\mathbf{m}^{\mathrm{c}} \mathbf{n}^{\mathrm{c}}\right): \frac{\partial^{2} W\left(\mathbf{F}_{\mathrm{c}}\right)}{\partial \mathbf{F} \partial \mathbf{F}}:\left(\mathbf{m}^{\mathrm{c}} \mathbf{n}^{\mathrm{c}}\right)=\frac{\partial^{2} W\left(\mathbf{F}_{\mathrm{c}}\right)}{\partial \mathrm{F}_{i j} \partial F_{k l}} m_{i}^{\mathrm{c}} n_{i}^{\mathrm{c}} m_{k}^{\mathrm{c}} n_{l}^{\mathrm{c}}=0, \quad \mathbf{m}^{\mathrm{c}}, \mathbf{n}^{\mathrm{c}} \in \mathbb{R}^{3} ; \\
& \left\|\mathbf{m}^{\mathrm{c}}\right\|=\left\|\mathbf{n}^{\mathrm{c}}\right\|=1 .
\end{aligned}
$$

This feature is responsible for the existence of discontinuous strain gradients in the equilibrium solutions of elastic solids whose strain energy density is the macroscopic energy density $W(\mathbf{F})$, i.e. when the size of the microstructure effects are ignored. It is also the reason for the existence of localized strain equilibrium solutions, polarized perpendicular to $\mathbf{n}^{\mathrm{c}}$, i.c. $\mathbf{u}(\mathbf{X})=\mathbf{m}^{\mathrm{c}} g\left(\mathbf{n}^{\mathrm{c}} \cdot \mathbf{X}\right)$, where $g(z)$ is a smooth scalar function of its real argument $z$, for elastic solids whose strain energy density is given by $\widehat{W}(\mathbf{F}, \mathbf{G})$, i.e. when the size of microstructure effects are taken into account (see TRIANTAFYLLIDIS and AIFANTIS, 1986).

(ii) A quadratic in $\mathbf{G}$ energy correction term $\left(\varepsilon^{2} / 2\right) \mathbf{G}: \mathbf{B}(\mathbf{F})$ : $\mathbf{G}$ that accounts for the effects of microstructure and restores the strong ellipticity of the equilibrium equations for $\hat{W}$.

For a given microstructure there is no guarantee that the strong ellipticity condition for $\widehat{W}$, i.e.

$$
(\mathbf{m n n}) \vdots \mathbf{B}(\mathbf{F}) \vdots(\mathbf{m n n})>0 \quad \forall \mathbf{m}, \mathbf{n} \in \mathbb{R}^{3} ; \quad\|\mathbf{m}\|=\|\mathbf{n}\|=1,
$$

will be satisfied for any deformation gradient $\mathbf{F}$, unlike the recent stability inves-

\footnotetext{
+ Note: frame invariance requires $W(F)$ to be independent of rigid body rotations, in which case $\mathbf{I}$ can be more accuratcly replaced by an arbitrary rigid body rotation $\mathbf{R}$.
} 
tigations of shear bands by LeROY and MolinARI (1993) where a $\mathbf{B}(\mathbf{F})$ was postulated $a b$ initio so as to satisfy the strong ellipticity of $\widehat{W}$ for all $\mathbf{F}$. One has thus to check if there exists a neighborhood about the critical deformation gradient $\mathbf{F}_{\mathrm{c}}$ for which the strong ellipticity condition for $\widehat{W}(\mathbf{F}, \mathbf{G})$ holds, namely

$$
\begin{gathered}
(\mathbf{m n n}): \\
: \mathbf{B}\left(\mathbf{F}_{\mathrm{c}}+\Delta \mathbf{F}\right):(\mathbf{m m n})=B_{i j k l m n}\left(\mathbf{F}_{\mathrm{c}}+\Delta \mathbf{F}\right) m_{i} m_{l} n_{j} n_{k} n_{m} n_{n}>0 \\
\forall \mathbf{m}, \mathbf{n} \in \mathbb{R}^{3}, \quad\|\mathbf{m}\|=\|\mathbf{n}\|=1 ; \quad \forall\|\Delta \mathbf{F}\|<\delta,
\end{gathered}
$$

where the norm of the second rank tensor $\Delta \mathbf{F}$ is defined by $\|\Delta \mathbf{F}\|=(\Delta \mathbf{F}: \Delta \mathbf{F})^{1 / 2}=$ $\left(\Delta F_{i j} \Delta F_{i j}\right)^{1 / 2}$ and where $\delta \geqslant 0$. If a strictly positive $\delta$ can be found that satisfies (2.21) one can easily show, following the steps in TRIANTafYllidis and Aifantis (1986) that a "bell shaped" localized deformation polarized equilibrium solution [of the type $\mathbf{u}(\mathbf{X})=\mathbf{m}^{\mathrm{c}} g\left(\mathbf{n}^{\mathrm{c}} \cdot \mathbf{X}\right)$ ] can be constructed, as long as the deformation gradient lies at all points $\mathbf{X}$ within the above mentioned neighborhood of $\mathbf{F}_{\mathrm{c}}$. Failure of (2.21) indicates the non-existence of such smooth localized deformation equilibrium solutions since the admissible equilibrium solutions can now have discontinuities in $\mathbf{G}=\mathbf{F} \nabla$.

There is an important connection between the strong ellipticity (or lack of it) of $W(\mathbf{F})$ and $\widehat{W}(\mathbf{F}, \mathbf{G})$ and the stability of an arbitrary region of the continuum (assumed homogeneous) under constant strain and for full Dirichlet boundary conditions. To this end consider a volume $V$, with boundary $\partial V$, of a homogeneous continuum whose strain energy density is given by $\widehat{W}(\mathbf{F}, \mathbf{G})$ in (2.7). On the boundary $\partial V$ a displacement $\mathbf{u}=(\mathbf{F}(\lambda)-\mathbf{I}) \cdot \mathbf{X}$ is applied, where the deformation gradient $\mathbf{F}(\lambda)$ depends on a scalar parameter $\lambda \geqslant 0$, also termed the loading parameter, with $\mathbf{F}(0)=\mathbf{I}$. It is also assumed that the strain induced by $\mathbf{F}$ is a monotonically increasing function of $\lambda$, and this requirement is satisfied when the principal stretches $\dot{\lambda}_{i}(\lambda)$ of $\mathbf{F}$ (the eigenvalues of the pure stretch $\mathbf{U}$ part of $\mathbf{F}$ obtained from the polar decomposition $\mathbf{F}=\mathbf{R} \cdot \mathbf{U}$ where $\mathbf{R}$ is the rigid body rotation part of $\mathbf{F}$ ) increase or decrease monotonically away from unity, since $\lambda_{i}(0)=1$. As a result of the assumed homogeneity of the solid, an obvious solution to the equilibrium equations (2.17), is $\mathbf{u}=\mathbf{u}(\mathbf{X}, \lambda)=(\mathbf{F}(\lambda)-\mathbf{I}) \cdot \mathbf{X}, \forall \mathbf{X} \in V$, which implies uniform strain in $V$, i.e. $\mathbf{F}=\mathbf{F}(\hat{\lambda}), \mathbf{G}=\mathbf{F} \nabla=\mathbf{0}, \forall \mathbf{X} \in V$. Of interest is the stability of the above found uniform strain solution under arbitrary perturbations $\delta \mathbf{u}(\mathbf{X})$ satisfying $\delta \mathbf{u}=\mathbf{0}, \delta \mathbf{u} D=0$ (which also imply $\delta \mathbf{u} \nabla=\mathbf{0}$ ), for $\mathbf{X} \in \partial V$ in view of the assumed imposition of displacement and its normal derivative at the boundary.

Using the minimum potential energy criterion, the uniform strain solution is stable if it corresponds to a local minimum of the solid's total energy $\mathscr{E}=\int_{V} \widehat{W} \mathrm{~d} V$. Given that $\widehat{W}$ is a smooth function of $\mathbf{u}$ the local minimum condition can be written as $\left(\mathscr{E}_{\text {.uu }}(\mathbf{u}(\lambda)) \cdot \delta \mathbf{u}\right) \cdot \delta \mathbf{u}>0 \forall \delta \mathbf{u} \neq 0$ where $\delta \mathbf{u}$ is a kinematically admissible displacement (i.e. $\delta \mathbf{u}$ is an adequately smooth function with continuous gradient $\delta \mathbf{u} \nabla$ and $\delta \mathbf{u}=\delta \mathbf{u} \nabla=\mathbf{0}$ on $\partial V)$ and $\mathscr{E}{ }_{\text {uu }}(\dot{\mathbf{u}}(\hat{\lambda}))$ is the second Frechet derivative of the energy $\mathscr{E}$ evaluated at the uniform strain solution. Hence, from (2.7) the uniform strain solution $\mathbf{F}(\lambda)$ is stable if for all $\delta \mathbf{u} \neq \mathbf{0}$ :

$$
\begin{aligned}
\left(\mathscr{E}_{\mathbf{u u}}(\mathbf{u}) \cdot \delta \mathbf{u}\right) \cdot \delta \mathbf{u}=\int_{V}\left[(\delta \mathbf{u} \nabla): \frac{\partial^{2} W(\mathbf{F}(\lambda))}{\partial \mathbf{F} \partial \mathbf{F}}:(\delta \mathbf{u} \nabla)\right. & \\
& \left.+\varepsilon^{2}((\delta \mathbf{u} \nabla) \nabla): \mathbf{B}(\mathbf{F}(\lambda)):((\delta \mathbf{u} \nabla) \nabla)\right] \mathrm{d} V>0 .
\end{aligned}
$$


Notice that for a homogeneous body and a miform strain equibribm freld $\partial^{2} W(\mathbf{F}(\lambda)) / \partial \mathbf{F} \partial \mathbf{F}$ and $\mathbf{B}(\mathbf{F}(\lambda))$ are constant tensors. Moreover observe that the Fourier transform of $\delta \mathbf{n}$ exists in the classical sense since $\delta \mathbf{u}$ is a contimuous function in $\mathbb{R}^{3}$ with compact support ( $\delta \mathbf{u}$ can be extended into the entire space $\mathbb{R}^{3}$ by taking $\delta \mathbf{u}=\mathbf{0}$ for $\mathrm{X} \in \mathbb{R}^{3} \backslash V$ ). Hence by making use of the inner product preserving property of the Fourier transform, $(2.22)$ can be equivalently rewritten as:

$$
\int\left[(\delta \mathbf{C} \Xi): \frac{\partial^{2} W(\mathbf{F}(\lambda))}{\partial \mathbf{F} \partial \mathbf{F}}:(\delta \mathbf{U} \Xi)+\partial^{2}(\delta \mathbf{U} \Xi \Xi): \mathbf{B}(\mathbf{F}(\lambda)):(\delta \mathbf{U} \Xi \mathbf{E})\right] \mathrm{d} V=0
$$

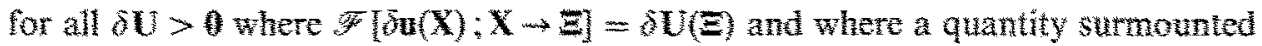
by a bar ( ) denotes the complex conjugate of the quantity in question

The arbitrariness of $\delta(\mathbf{X})$ and hence $\delta U(\Xi)$ imply that $(2.23)$ is satisfed if the second rank tensor $\mathrm{S}(\boldsymbol{F}(\lambda), \Xi)$ is positive definite, i.e.

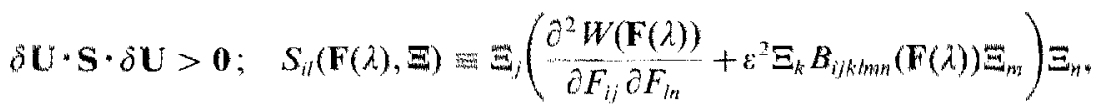

where, without loss of generality, $\delta \mathbf{U}, \equiv$ are arbitrary unit vectors in $\| \mathbb{R}^{3}(\|\delta \mathbf{U}\|=$ $\|\equiv\|=1$ ). Stability of the uniform strain solution $\mathbf{F}(\lambda)$ under Dirichlet boundary conditions is thus assured when $\beta_{S}(\mathbf{F}(\lambda))>0$, where $\beta_{S}(\mathrm{~F}(\lambda))$ is the mininum eigenvalue of $\mathbf{S}\left(\mathbf{F}(\beta)\right.$, E) for all possible unit pectors $\mathrm{E}$. Since $\beta_{s}(\boldsymbol{F}(\lambda))=$

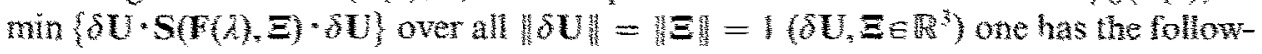
ing inequality:

$$
\begin{aligned}
& \beta_{s}(\mathbf{F}(\lambda)) \geqslant \beta_{x}(\boldsymbol{F}(\lambda))+\varepsilon^{3} \hat{\beta}_{B}(\mathbf{F}(\lambda)) \\
& \left.\beta_{S}(\mathbf{F}(\lambda))=\min _{\delta(t)}\{\delta \mathbf{U} \cdot \mathbf{S}(\mathbf{F}(\lambda)), \mathbf{\Xi}) \cdot \delta \mathbf{U}\right\} \text {, } \\
& \beta_{L}(\mathbf{F}(\lambda))=\min _{\delta L, \Xi}\left\{(\delta \mathbf{U} \boldsymbol{\Xi}): \frac{\partial^{2} W(\mathbf{F}(\lambda))}{\partial \mathbf{F} \partial \mathbf{F}}:(\delta \mathbf{U} \boldsymbol{\Xi})\right\},
\end{aligned}
$$

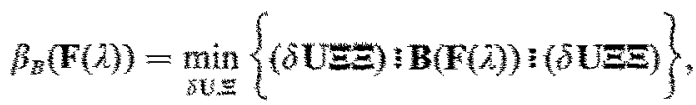

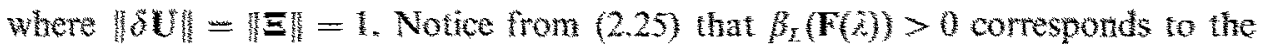
strong ellipticity of macroscopic energy density $W(F)$ at $F(\lambda)$ (see also discussion of $(2.20)]$ since it ensures the positive defunteness of the tensor with components $\sum_{j}\left(\partial^{2} W(\mathbf{F}(\lambda)) / \partial F_{i j} \partial F_{k l}\right) \Xi_{i}$, while $\beta_{\beta}(\mathbf{F}(\lambda))>0$ corresponds to the strong ellipticity of $W(\mathbf{F}, \mathbf{G})$ at $\mathbf{F}(\lambda)$ [see also discussion of $(2.21)$ ] since it ensures the positive definiteness of the tensor with components $\Xi_{j} \Xi_{k} B_{i j k i m n}(\mathbf{F}(\lambda)) \Xi_{n} \Xi_{n}$.

Based on this observation, the following conclusions can be drawn about the stability of the uniform strain solution $F(\lambda)$ under displacement controlled boundary conditions in a homogeneous elastic body whose energy density $W(F, G)$ is given by (2.7). As the wiform strain in the sold increases and for as longas $W(\mathrm{~F}(\mathrm{x}))$ is strongly

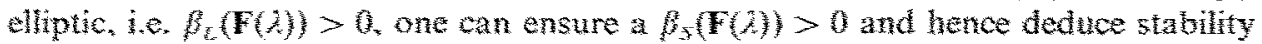


for the uniform strain problem under displacement control. If $\beta_{B}(\mathrm{~F}(\hat{\lambda}))>0$ then any size part of the solid under $\mathbf{F}(\lambda)$ is stable, whether for $\beta_{B}(\mathbf{F}(\lambda))<0$ one has to consider an adequately small value of $\varepsilon$, or equivalently a large sample--compared to the size of the microstructure-in order to ensure $\beta_{S}(\mathbf{F}(\lambda))>0$. When during the above loading process $\lambda$ reaches a critical value $\lambda_{\mathrm{c}}$ corresponding to the loss of ellipticity of $W$, at $\mathbf{F}_{\mathrm{c}}=\mathbf{F}\left(\lambda_{\mathrm{c}}\right)$ and hence $\beta_{L}\left(\mathbf{F}_{\mathrm{c}}\right)=0$ [see also $(2.20)_{2}$ ], the corresponding uniform strain solution is stable only if $\widehat{W}$ is strongly elliptic at $\mathbf{F}_{\mathrm{c}}$, i.e. $\beta_{B}\left(\mathbf{F}_{\mathrm{c}}\right)>0$. Consequently one can deduce that as long as $\widehat{W}$ is strongly elliptic at $\mathbf{F}_{\mathrm{c}}$ and (2.21) is satisfied [which would imply $\beta_{B}\left(\mathbf{F}_{\mathrm{c}}\right)>0$ ], a uniform strain critical deformation $\mathbf{F}_{\mathrm{c}}$ can be reached and sustained, in view of its stability via a displacement control process in a homogeneous elastic solid whose energy density $\widehat{W}(\mathbf{F}, \mathbf{G})$ is given by $(2.7)$. When $\beta_{B}\left(\mathbf{F}_{\mathrm{c}}\right)<0$ one can show that the uniform critical deformation $\mathbf{F}_{\mathrm{c}}$ corresponding to the loss of ellipticity of $W(\mathbf{F})$ cannot be reached and that some bifurcation type instability will develop during the loading process corresponding to $\mathbf{F}(\lambda)$ for some $\lambda<\lambda_{\mathrm{c}}$ (the exact value of $\lambda$ depending on the size of the solid), since $\mathbf{F}_{\mathrm{c}}$ is not a local minimizer of $\widehat{W}$.

\section{EXAmples for SPeCific Microstructures}

As previously mentioned, all investigations to date of localized deformations using higher order gradient models for non-linear elastic continua, postulate an energy density $\widehat{W}(\mathbf{F}, \mathbf{G})$, as given by $(2.7)$, for which $\mathbf{B}(\mathbf{F})$ satisfies the strong ellipticity requirement (2.21). In contrast with the previously mentioned work, the energy density $\widehat{W}(\mathbf{F}, \mathbf{G})$ is now calculated from the microstructure, i.e. from lattice geometry and interaction potentials. Consequently the ellipticity of $\mathbf{B}(\mathbf{F})$ must be checked for all $\mathbf{F}$ near critical deformations $\mathbf{F}_{\mathrm{c}}$ as indicated in (2.21). In this section the energy densities corresponding to two different two-dimensional microstructures, each being symmetric with respect to the $X_{1}$ and $X_{2}$ axes, are investigated.

The first microstructure, hereafter referred to as Case $\mathrm{A}$, is a two-dimensional extension of the one-dimensional non-linear elastic model used in TRIANTAFYLLIDIS and BARDENHAGEN (1993). The Case A microstructure is a square lattice whose unit cell is depicted in Fig. 2. The unit cell consists of non-linear springs connecting the center node, depicted in white, to nodes in each of the first two surrounding squares. depicted in black. It will be shown that the Case A model is an example of a microstructure for which the continuum energy density $\widehat{W}$ satisfies the strong ellipticity requirement (2.21), and for which localized strain solutions are possible.

The second microstructure, hereafter referred to as Case $\mathrm{B}$, is motivated by our desire to study continuum models of monatomic (i.e. composed of only one atomic species), perfect crystals. The Case B microstructure consists of a hexagonal lattice with a node at each lattice site as depicted in Fig. 3(a). The center node of each unit cell is again depicted in white while all the other nodes interacting with it are depicted in black. The nodal interaction potentials in Case B are identical for each node and have range $L$, i.e. $\phi_{N}\left(l_{N}\right)=\phi\left(l_{N}\right)$ for $L_{N} \leqslant L$ and $\phi_{N}\left(l_{N}\right)=0$ for $L_{N}>L$. The effect on the ellipticity of $\mathbf{B}(\boldsymbol{F})$ of increasing the potential's range $L$ from $L=\varepsilon$ (only six 


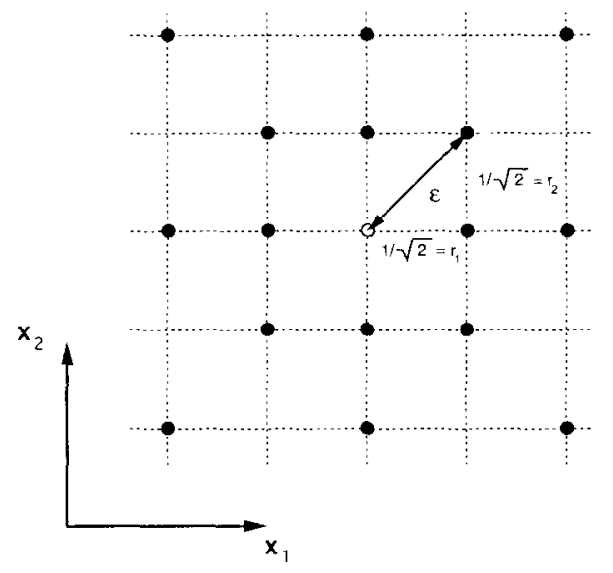

Fig. 2. The unit cell for the Case A microstructure. The grid spacings are $\sqrt{2}$ in the $X_{1}$ and $X_{2}$ directions. The center nodc, which is depicted in white, is connected by non-linear springs to nodes in the first two surrounding scuuares, depicted in black.

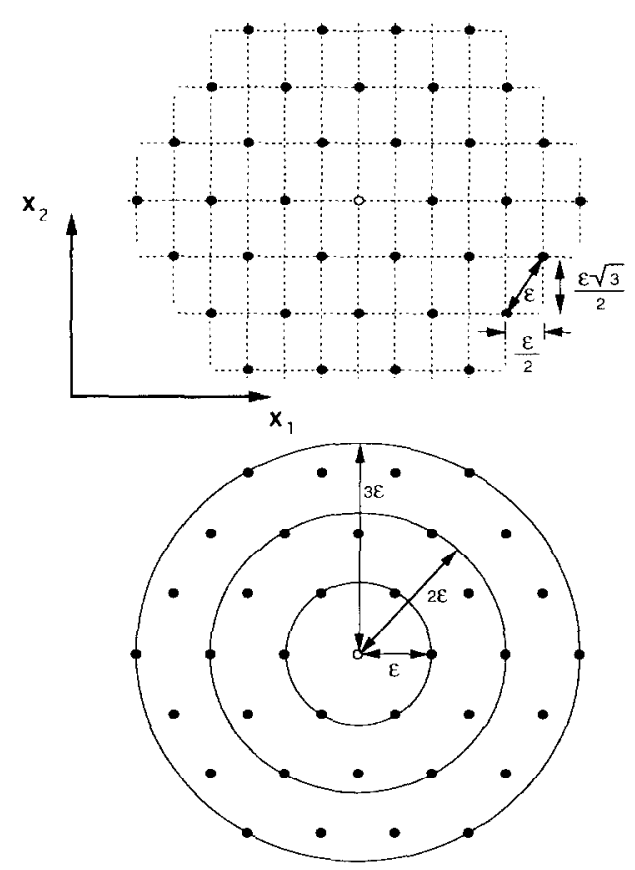

Fig. 3. The unit cell for the Case B microstructure. The grid spacings are $8 / 2$ in the $X_{1}$ direction and $\varepsilon \sqrt{3} / 2$ in the $X_{2}$ direction. The center node, depicted in white, interacts in accordance with an atomic potential with nodes in surrounding hexagons, depicted in black. (a) The hexagonal lattice. superimposed on the grid. (h) Illustration of the unit cell center node and its 36 nearest neighbors. 
nearest nodes interact with the center node) to $L=2 \varepsilon$ ( 18 nearest nodes interact with the center node) and finally to $L=3 \varepsilon$ (36 nodes interact with the center node) as depicted in Fig. 3(b) is studied.

Of interest is the determination of the critical deformation gradient, $\mathbf{F}_{\mathrm{c}}$, at which the macroscopic energy density $W(\mathbf{F})$ loses strong ellipticity and the size of the region, $\delta$, about $\mathbf{F}_{c}$ for which $\mathbf{B}(\mathbf{F})$ maintains strong ellipticity of $\widehat{W}(\mathbf{F}, \mathbf{G})$, according to (2.21). For monotonically increasing uniform strain principal solutions $\mathbf{F}=\mathbf{F}(\lambda)$, $\mathbf{G}=\mathbf{F} \nabla=\mathbf{0}$, as discussed in Section 2.4 [where $\lambda \geqslant 0$ is the loading parameter and $\mathbf{F}(0)=\mathbf{I}], \mathbf{F}_{\mathrm{c}}$ and $\delta$ are found by investigating the positive definiteness of the following $2 \times 2$ matrices $\left[A_{i k}\right]$ and $\left[B_{i i}\right]$ :

$$
A_{i k}(\lambda, \mathbf{n})=\frac{\partial^{2} W(\mathbf{F}(\lambda))}{\partial F_{i j}} \frac{\partial F_{k l}}{n_{j}} n_{l}, \quad B_{i l}(\lambda, \mathbf{n})=B_{i j k l m n}(\mathbf{F}(\lambda)) n_{j} n_{k} n_{m} n_{n} .
$$

The loss of strong ellipticity for $W(\mathbf{F})$ and $\widehat{W}(\mathbf{F}, \mathbf{G})$ corresponds to loss of positive definiteness for $\left[A_{i k}\right]$ and $\left[B_{i l}\right]$ respectively.

In two dimensions positive definiteness of $\left[A_{i k}\right]$ and $\left[B_{i i}\right]$ is determined by examining the real roots of certain polynomials in $n_{2} / n_{1}$ which are the principal minors of $\left[A_{i k}\right]$ and $\left[B_{i i}\right]$. More specifically, the principal minors of $\left[A_{i k}\right]$ are $A_{i i}$ (no sum) and $\operatorname{det}\left[A_{i k}\right]$ which are second and fourth order polynomials in $n_{2} / n_{1}$ respectively, with coefficients depending on the loading parameter $\lambda$. Similarly the principal minors of $\left[B_{i i}\right]$ are $B_{i i}$ (no sum) and det $\left[B_{i l}\right]$ which are fourth and eighth order polynomials in $n_{2} / n_{1}$ respectively, with coefficients depending on the loading parameter $\lambda$. Real roots of these polynomials are sought since their existence implies a change of sign and hence loss of positive definiteness in the corresponding matrices. To determine the number of distinct real roots of a real $n$th order polynomial $p_{n}(x)$, it is convenient to compute the Cauchy index of the function $p_{n}^{\prime}(x) / p_{n}(x)$ in the entire real line, denoted by $I_{-x}^{+\infty}\left[p_{n}^{\prime}(x) / p_{n}(x)\right]$, by constructing a Sturm chain (see GanTMaCHeR, 1974). For completeness of the presentation this procedure is briefly outlined in the Appendix.

Results of ellipticity calculations for Cases $A$ and $B$ are presented for uniform strain deformations having the $X_{1}$ and $X_{2}$ directions as principal directions, i.e.

$$
\mathbf{F}(\lambda)=\left[\begin{array}{cc}
\lambda_{1}(\lambda) & 0 \\
0 & \lambda_{2}(\lambda)
\end{array}\right]
$$

This class of deformations is sufficient to completely characterize all uniform strain states for isotropic materials. Only the Case B microstructure under infinitesimal deformations is isotropic. The same microstructure under large strains as well as the Case A microstructure produce macroscopic energy densities $W(\mathbf{F})$ which are orthotropic with equivalent orthotropy axes $X_{1}, X_{2}$.

Two graphs in the $\lambda_{1}, \lambda_{2}$ plane are produced for the ellipticity results of each microstructure discussed. The first graph presents the ellipticity domain of the macroscopic energy density $W(\mathbf{F})$ [where (2.20), holds] for the uniform strain deformations $\mathbf{F}(\hat{\lambda})$ given by (3.1). Strong ellipticity for deformation gradients $\mathbf{F}(\lambda)$ along a particular path $\left(\lambda_{1} / \lambda_{2}=\right.$ const $)$ is indicated by a solid line.

The second graph presents results from the ellipticity investigation for $\widehat{W}(\mathbf{F}, \mathbf{G})$, or equivalently for $\mathbf{B}(\mathbf{F})$, for several different uniform strain paths $\mathbf{F}(\lambda)$. The paths 
correspond to stretching in the $X_{1}$ and $X_{2}$ directions $\left(\lambda_{1}=\lambda_{2} \lambda_{2}=1\right.$, and $\lambda_{1}=1$, $\lambda_{2}=\lambda$, respectively), balanced biaxial stretching $\left(\lambda_{1}=\lambda_{2}=\lambda\right)$, and isochoric deformation $\left(\lambda_{1}=\lambda_{2} \lambda_{2}=1 / \lambda_{2}\right)$. Strong ellipticity of the macroscopic energy density $W(\mathbf{F})$ along these deformation paths is indicated by a solid line and the angle between vector $\mathbf{n}^{c}$ and the $X_{1}$-axis [where $(2.20)_{2}$ holds, indicating the initial orientation of shear localization] is presented. For microstructures whose continuum energies $\hat{W}(\mathbf{F}, \mathbf{G})$ satisfy the strong ellipticity requirement [(2.21) holds for all $\|\Delta \mathbf{F}\|<\delta]$, the corresponding maximal value of $\delta$ is also given on the same graph.

Case $A$

For Case A the microstructure is a square lattice $\left(r_{1}=r_{2}=1 / \sqrt{2}\right)$ with nodes at each lattice site and potentials derived from non-linear springs, which are stress free at the reference state $\mathbf{u}=\mathbf{0}$. Each node is connected to all nodes on the nearest surrounding square by non-linear springs which have dimensionless potentials $w_{N}\left(\beta_{N}\right)=w_{1}\left(\beta_{N}\right)$ and to selected nodes on the outer surrounding square by non-linear springs with dimensionless polentials $w_{N}\left(\beta_{N}\right)=w_{2}\left(\beta_{N}\right)$ (as seen in Fig. 2 ), where

$$
w_{i}\left(\beta_{N}\right)=\frac{1}{2} K_{i} \beta_{N}^{2}-\frac{1}{4} M_{i} \beta_{N}^{4}+\frac{1}{6} N_{i} \beta_{N}^{6} .
$$

The common modulus $E$ in the definition (2.4) of $\phi_{N}$ is taken to be $E=1$. The spring constants chosen for this case are

$$
\begin{array}{lll}
K_{1}=1, & M_{1}=8, & N_{1}=20, \\
K_{2}=-1 / 2, & M_{2}=5, & N_{2}=-10 .
\end{array}
$$

The dimensionless spring force $w_{1}^{\prime}\left(\beta_{N}\right), w_{2}^{\prime}\left(\beta_{N}\right)$ vs strain $\beta_{N}$ relations are plotted in Fig. 4 . The dimensionless potentials are characterized by the properties $w_{1}^{\prime}\left(\beta_{\lambda}\right) \rightarrow \infty$ as $\beta_{X} \rightarrow \infty$ for the interaction of the center node with the nodes on the nearest surrounding square, and $w_{2}^{\prime}\left(\beta_{N}\right) \rightarrow-\infty$ as $\beta_{N} \rightarrow \infty$ for the interactions of the center node with the nodes on the outer surrounding square. The negative slope springs connecting the center node to the nodes on the outer surrounding squares are responsible for $\mathbf{B}(\mathbf{F})$ satisfying strong ellipticity requirement $(2.21)$ in analogy to the onedimensional result in TRIANTAFYLLIDIS and BARDENHAGEN (1993).

The continuum encrgy density functions $W(\mathbf{F})$ and $\mathbf{B}(\mathbf{F})$ have been calculated by using (3.2) in (2.11). The ellipticity domain of macroscopic energy density $W(\mathbf{F})$ for all deformation gradients $\mathbf{F}(\lambda)$ with principal axes $X_{1}, X_{2}$, as given by (3.1), is presented in Fig. 5. Each line corresponds to a fixed ratio of $\left(\lambda_{1}-1\right) /\left(\lambda_{2}-1\right)$ and is terminated at the critical deformation gradient $\mathbf{F}_{\mathrm{c}}=\operatorname{diag}\left[\lambda_{1 \mathrm{c}}, \lambda_{2 \mathrm{c}}\right]$ at which strong ellipticity is lost, i.e. $(2.20)_{2}$ holds. The ellipticity domain is symmetric about the balanced biaxial stretching path $\lambda_{1}=\lambda_{2}=\lambda$ as expected from a discrete microstructure which is symmetric with respect to the $X_{1}=X_{2}$ line, or invariant under a $\pi / 2$ rotation.

For the four loading paths mentioned in the general discussion at the beginning of this section, i.e. unidirectional stretching in the $X_{1}$ and $X_{2}$ directions. balanced biaxial stretching, and isochoric deformation, the ellipticity results for $\widehat{W}(\mathbf{F}, \mathbf{G})$ are presented in Fig. 6. Solid lines are terminated at the critical deformation gradient $\mathbf{F}_{\mathrm{c}}=\operatorname{diag}\left[\hat{\lambda}_{k,}, \hat{\lambda}_{2 \mathrm{c}}\right]$ at which strong ellipticity of the macroscopic energy density $W(\mathbf{F})$ 

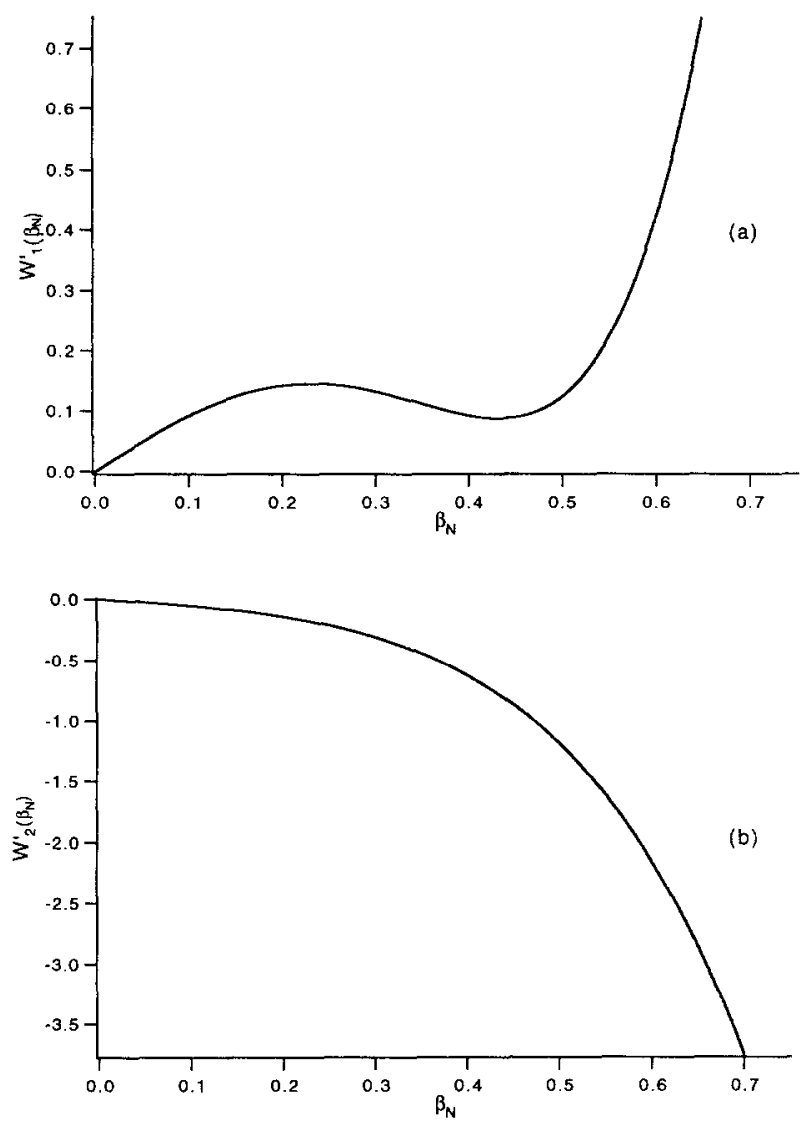

FIG. 4. Force displacement profiles for Case A microstructure. (a) For non-linear springs connecting each center node to nodes in the first surrounding square. (b) For non-linear springs connecting each center node to nodes in the second surrounding square.

is lost, while $\theta$ denotes the angle between $\mathbf{n}^{\mathrm{c}}$ and the $X_{1}$ axis, which determines the initial orientation of the localized strain zone. Moreover $\delta$ denotes the norm of $\Delta \mathbf{F}$, which determines the size of the region about $\mathbf{F}_{\mathrm{c}}$ for which $\widehat{W}(\mathbf{F}, \mathbf{G})$ is strongly elliptic according to (2.21).

The ellipticity results for $\widehat{W}(\mathbf{F}, \mathbf{G})$ are symmetric about the balanced biaxial stretching path as in Fig. 5 due to the symmetry of the lattice, as previously explained. Along this path no direction is preferred for the localized deformation zone due to the symmetry of the microstructure and the symmetry of the loading. For each path a finite neighborhood about $\mathbf{F}_{\mathrm{c}}$ exists, i.e. $\delta>0$, for which $\widehat{W}(\mathbf{F}, \mathbf{G})$ is strongly elliptic. Note that $\delta$ increases monotonically with the orientation of deformation angle $\psi, \tan \psi=\left(\lambda_{2}-1\right) /\left(\lambda_{1}-1\right)$, as $\psi$ moves from $-\pi / 4$ (balanced biaxial compression) to $\pi / 4$ (balanced biaxial tension). The microstructure of Case $\mathrm{A}$ thus gives rise to a continuum model which satisfies the properties necessary for the existence of localized strain solutions, polarized along the critical direction indicated at the end of each corresponding strain path in Fig. 6. 


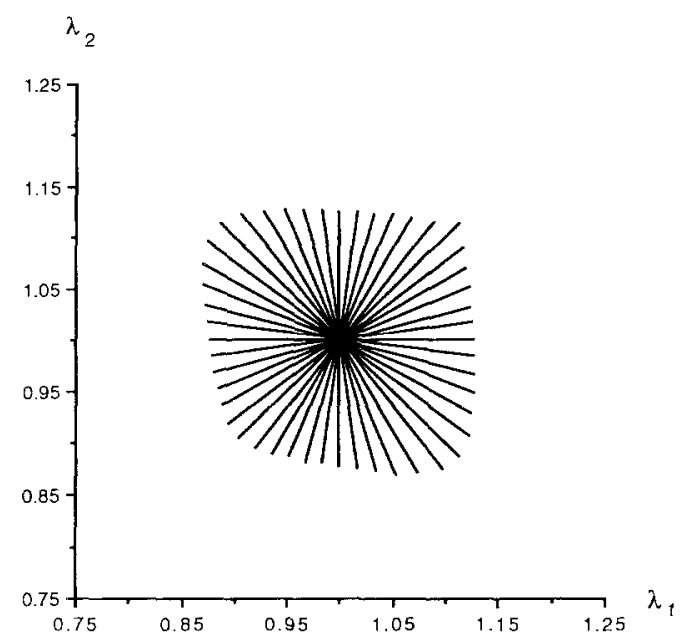

Fig. 5. Elliplicity domain of $W(\mathbf{F})$ for Case $A$ microstructure in $\lambda_{1} i_{2}$ space, where $\lambda_{1}, \lambda_{2}$ are the principal stretches. Each solid line corresponds to a fixed ratio of $\left(\lambda_{1}-1\right)\left(\lambda_{2}-1\right)$ and is terminated at the point at which strong ellipticity of $W(\mathbf{F})$ is lost.

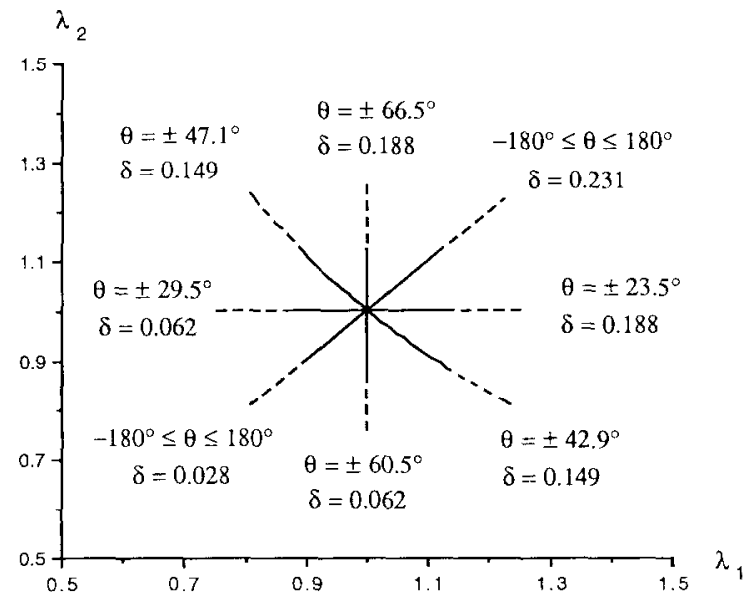

Fig. 6. Ellipticity results for $\mathbf{B}(\mathbf{F})$ for Case $A$ mierostructure in $\lambda_{1}-\lambda_{2}$ space, where $\lambda_{1}, \lambda_{2}$ are the principal stretches. Fach solid line corresponds to a particular loading path (see Section 3. Case A) and is terminated at the point at which strong ellipticity of $W(\mathbf{F})$ is lost. The angle $\theta$ indicates the initial orientation of the localized strain zone, and $\delta$ indicates the size of the region about $\mathbf{F}_{\mathrm{c}}$ for which $\mathbf{B}(\mathbf{F})$ is strongly elliptic.

\section{Case B}

For Case $\mathrm{B}$ the microstructure is a hexagonal lattice $\left(r_{1}=1 / 2, r_{2}=\sqrt{3} / 2\right)$ with nodes at each lattice site as depicted in Fig. 3(a). Each node has the same atomic potential. This model is motivated by our desire to study continuum models of monatomic (i.e. composed of only one atomic species), perfect crystals. Although a realistic crystal model should be three-dimensional, the verification of positive 
(a)

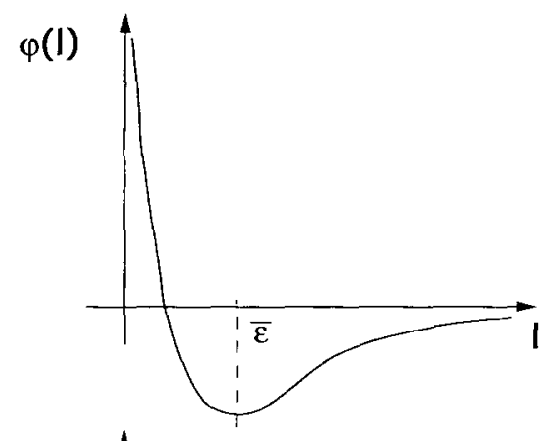

(b)

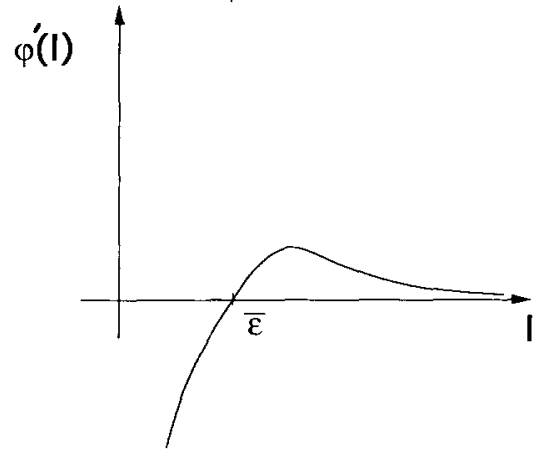

FIG. 7. Typical atomic interaction potential for the Case B microstructure. (a) Energy $\phi(l)$ of the interaction between the center node and a node at distance $l$ from the center node. (b) Central force $\phi^{\prime}(l)$ experienced at the center node due to the presence of a node at distance $l$.

definiteness of $\left[A_{i k}(\lambda, \mathbf{n})\right],\left[B_{i l}(\lambda, \mathbf{n})\right]$ leads to the examination of roots for polynomials of two variables $\left(n_{2} / n_{1}, n_{3} / n_{1}\right)$ for which no extension of the Sturm chain method exists. In the interest of simplicity our crystal model has thus been restricted to two dimensions.

A typical atomic potential $\phi(l)$ and its force $\phi^{\prime}(l)$ are depicted in Fig. 7. In accordance with standard models (see BORN and HUANG, 1954; WIENER, 1983), a strong repulsive force between atoms at close range, and a diminishing attractive force at long range dictates that $\phi^{\prime}(l) \rightarrow-\infty$ as $l \rightarrow 0$, and $\phi^{\prime}(l) \rightarrow 0$ as $l \rightarrow \infty$ respectively. The minimum value of $\phi(l)$ is attained at $l=\bar{\varepsilon}$, where the force $\phi^{\prime}(\bar{\varepsilon})=0$. It is seen that the force $\phi^{\prime}(l)$ decays to zero rapidly as $l \rightarrow \infty$ and thus the dominant interactions are between nearest neighbors. A perfect crystal with atomic potential $\phi(l)$ may then be approximated by taking into account only the interactions of nodes within a finite range $L$.

For Case $\mathrm{B}$, the dimensionless potential with range $L$ is given by

$$
\begin{aligned}
w_{N}\left(\beta_{N}\right) & =w\left(\beta_{N}\right)=\frac{\phi\left(l_{N}\right)}{r_{1} r_{2} \varepsilon^{2}} & \text { for } L_{N} \leqslant L, \\
& =\frac{\left.-\bar{\varepsilon}^{6} \frac{\bar{\varepsilon}^{6}}{\varepsilon^{6}\left(\Delta_{N k}\right.} \overline{\Delta_{N k}}\left(\overline{2 \beta_{N}}+1\right)\right)^{3}}{4}+\frac{3 \bar{\varepsilon}^{8}}{4 \varepsilon^{8}\left(\Delta _ { N k } \overline { \Delta } _ { N k } \left(2 \beta_{N}\right.\right.}+\overline{1))^{4}} & \\
w_{N}\left(\beta_{N}\right) & =0 & \text { for } L_{N}>L,
\end{aligned}
$$


where $L_{*}=\varepsilon\left(\Delta_{k k} \Delta_{N k}\right)^{12}[\sec (2.1)]$, where $\bar{s}$ is a constant of the model and where the common modulus $E$ in the definition (2.4) of $\phi_{V}$ is taken to be $E=1$. From (2.3) the relationship between $l_{N}$ and $\beta_{N}$ is seen to be $l_{N}=s\left[\Delta_{N k} \Delta_{N k}\left(2 \beta_{N}+1\right)\right]^{1 / 2}$ and $\phi\left(l_{N}\right)$ may easily be deduced from (3.3).

Results for three subcases, for which the interaction range $L$ is varied [see Fig. 3(b)], are presented. For subcase B1, interactions between nearest neighbors, i.e. only interactions between the center node and those in the nearest surrounding hexagon, are considered $(L=\varepsilon)$. This subcase represents the simplest approximation to a perfect crystal [in which all nodes have atomic potential $\phi(l)$, or dimensionless polential $\left.u\left(\beta_{y}\right)\right]$. For subcase $B 2$. interactions between the center node and the nearest 18 neighbors are considered ( $L=2 g$ ). For subcase B3, interactions between the center node and the nearest 36 neighbors are considered $(L=3 \theta)$.

Increasing the range $L$ of dimensionless potential $w\left(\beta_{\checkmark}\right)$. alters the energy density $\widehat{W}(\mathbf{F}, \mathbf{G})$ as can be seen from $(2.11)$ defining its construction from the discrete microstructure. (The summations are over more nodes.) In particular, the energy density of the undeformed configuration $\widehat{W}(\mathbf{I}, \mathbf{0})$ depends on the range of $w\left(\beta_{N}\right)$, the lattice geometry, and the grid spacing $\varepsilon$,

$$
\widehat{W}(\mathbf{I}, \mathbf{0})=\left.\frac{1}{2} \sum_{N} w\left(\beta_{N}\right)\right|_{\beta_{N}=0}=\frac{1}{2} \sum_{N}\left\{\begin{array}{c}
-\bar{\varepsilon}^{6} \\
\varepsilon^{6}\left(\Delta_{N i} \Delta_{N i}\right)^{3}
\end{array}+\frac{3 \bar{\varepsilon}^{8}}{4 \varepsilon^{8}\left(\Delta_{N i} \Delta_{N i}\right)^{4}}\right\} .
$$

Note that due to its symmetry, the lattice is in equilibrium for any grid spacing (the sum of all forces at each lattice site is zero). In addition to equilibrium, the ground state has to be stable, i.e. its energy $\widehat{W}(\mathbf{I}, \mathbf{0})$ as a function of the only free parameter in the model, the lattice distance $s$, has to be minimized. A calculation from $\mathrm{d} \widehat{W}(\mathbf{I}, \mathbf{0}) / \mathrm{d} \varepsilon=0$ yields for subcase $\mathrm{B} 1 \varepsilon=\bar{\varepsilon}$, for subcase $\mathrm{B} 2 \varepsilon=0.98255 \bar{\varepsilon}$, and for subcase B3 $s=0.97968 \bar{c}$. Note that the undeformed state is not stress free, in general, when the potential range is large enough to include more than nearest neighbors (see BORN and HUANG, 1954; WIENFr, 1983).

The continuum energy density functions $W(\mathbf{F})$ and $\widehat{W}(\mathbf{F}, \mathbf{G})$ have been calculated using (3.3) in (2.11). The ellipticity domain of macroscopic energy density $W(\mathbf{F})$ for all deformation gradients $\mathbf{F}(\lambda)$ with principal axes $X_{1}$ and $X_{2}$, as given by (3.1), is presented in Fig. 8 for the three subcases. Each line corresponds to a fixed ratio of $\left(\lambda_{1}-1\right) /\left(\lambda_{2}-1\right)$ and is terminated at the critical deformation gradient $\mathbf{F}_{\mathrm{c}}=\operatorname{diag}\left[\lambda_{1}, \lambda_{2 \mathrm{c}}\right]$ at which strong ellipticity is lost, i.e. $(2.20)_{2}$ holds. Subcases B2 and B3 are very similar, indicating convergence toward the results for a perfect crystal [in which each node has interaction potential $\phi(l)$, or dimensionless potential $\left.w_{N}\left(\beta_{N}\right)\right]$. The ellipticity domain is not symmetric about the balanced biaxial stretching path

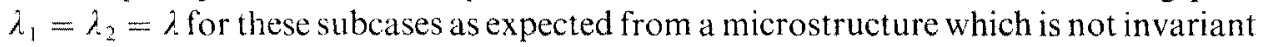
under a $\pi / 2$ rotation.

For the four loading paths, stretching in the $X_{1}$ and $X_{2}$ directions, balanced biaxial stretching, and isochoric deformation, the ellipticity results for $\widehat{W}(\mathbf{F}, \mathbf{G})$ are presented in Fig. 9 for the three subcases. Solid lines are terminated at the critical deformation gradient $\mathbf{F}_{0}=\operatorname{diag}\left[\lambda_{1}, \lambda_{x c}\right]$ at which strong ellipticity of macroscopic energy density $W(\mathbf{F})$ is lost, while $\theta$ denotes the angle between $\mathbf{n}$ and the $X_{1}$ axis, which determines the initial orientation of the localized strain zone. Along the balanced biaxial stretching 


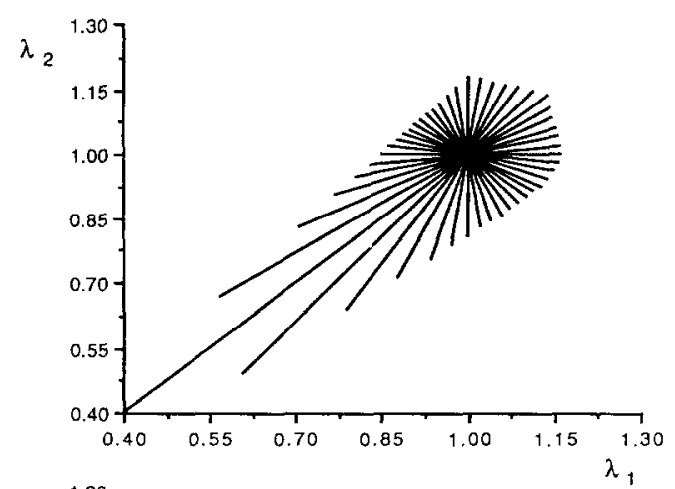

(a)

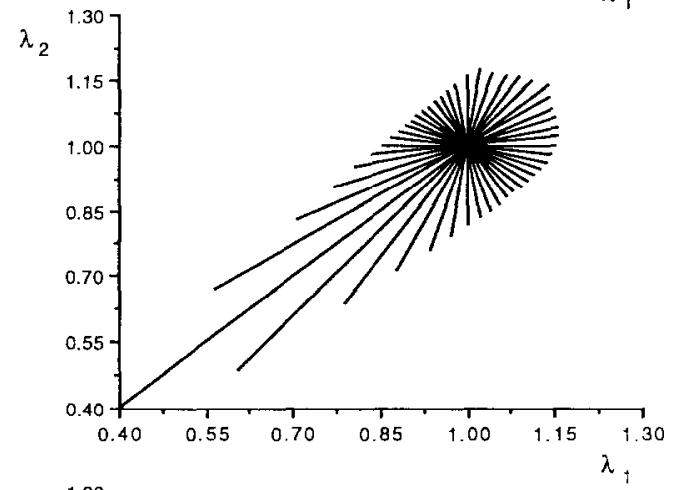

(b)

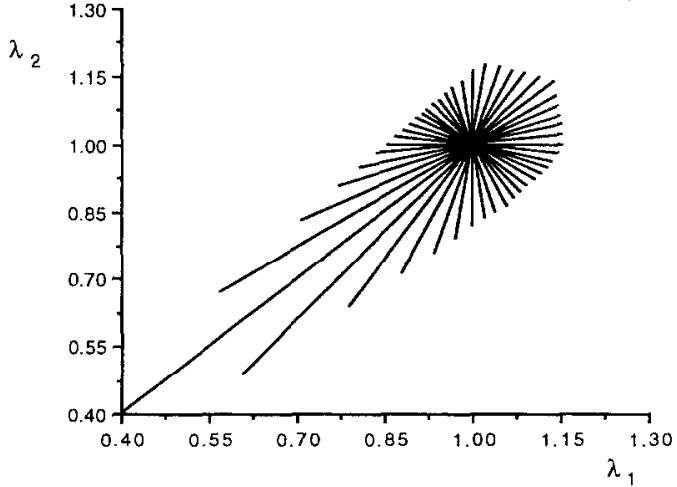

(c)

FIG. 8. Ellipticity domain of $W(F)$ for Case B microstructure in $\lambda_{1}-\lambda_{2}$ space, where $\lambda_{1}, \lambda_{2}$ are the principal stretches. Each solid line corresponds to a fixed ratio of $\left(\lambda_{1}-1\right) /\left(\lambda_{2}-1\right)$ and is terminated at the point at which strong ellipticity of $W(\mathbf{F})$ is lost. (a) For subcase BI only six nearest nodes interact. (b) For subcase B2 only 18 nearest nodes interact. (c) For subcase B3 only 36 nearest nodes interact.

path no direction is preferred for the localized deformation zone because the model is strained symmetrically along axes of symmetry. Ellipticity is not lost along the balanced biaxial stretching path in compression because this path is extremely stable as it takes infinite energy to bring two nodes to the same point (see Fig. 7).

For none of the subcases or loading paths is there a neighborhood about $\mathbf{F}_{\mathrm{c}}$ for 

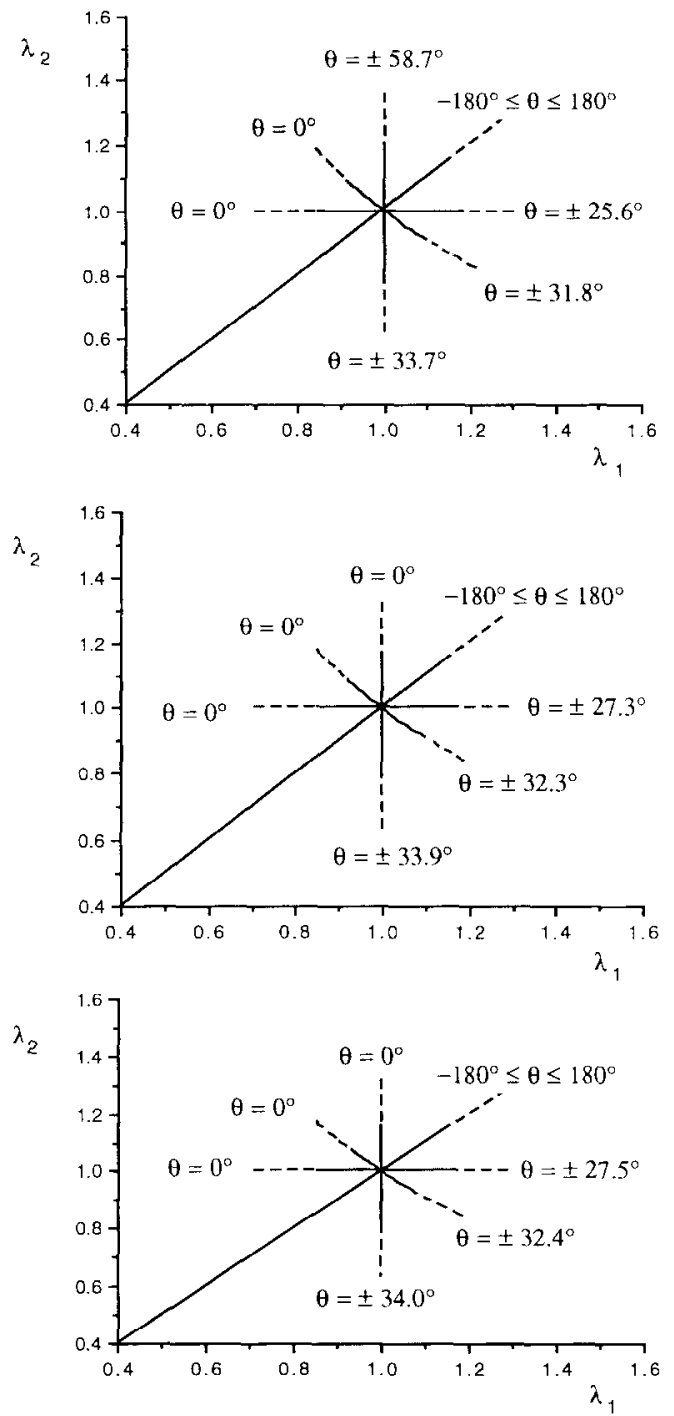

(c)

FIG. 9. Ellipticity results for $\mathbf{B}(\mathbf{F})$ for Case $\mathbf{B}$ microstructure in $\lambda_{1} \lambda_{2}$ space, where $\lambda_{1}, \lambda_{2}$ are the principal stretches. Each solid line corresponds to a particular loading path (see Section 3, Case B) and is terminated at the point at which strong ellipticity of $W(\mathbf{F})$ is lost. The angle $\theta$ indicates the initial orientation of the localized strain zone, and $\delta$ indicates the size of the region about $\mathbf{F}_{c}$ for which $\mathbf{B}(\mathbf{F})$ is strongly elliptic. (a) For subcase B1 only six nearest nodes interact. (b) For subcase B2 only 18 nearest nodes interact.

(c) For subcase B.3 only 36 nearest nodes interact.

which $\widehat{W}(\mathbf{F}, \mathbf{G})$ is strongly elliptic. Hence, according to the discussion in Section 2.4 . the microstructures become unstable on the principal path prior to reaching the critical deformation gradient $\mathbf{F}_{\mathrm{c}}$. This indicates that, for perfect crystals, some other bifurcated solution develops (not a localized strain solution) prior to the loss of ellipticity of 
macroscopic energy density $W(\mathbf{F})$. Indeed, bifurcation instabilities in crystals have already been reported in the literature at strain levels prior to maximum loads, a special case of loss of ellipticity of the first order gradient strain energy density [see for example THOMPSON and SHORROCK (1975) and references quoted therein].

\section{Discussion and Concluding Remarks}

The present study provides a consistent methodology for deriving non-linear higher order gradient continuum models from the properties of the underlying discrete periodic microstructures in three-dimensional non-linear elastic solids. The purpose of these models is to improve the continuum description of solids strained in their post-localization regime by including the scale effects of the underlying microstructure. This work is a generalization to three dimensions of the one-dimensional results in TRIANTAFYLLIDIS and BARDENHAGEN (1993) who initiated this type of calculation and compared the solutions of the discrete problem to the solutions of the corresponding continuum boundary value problem. In addition to their theoretical interest. an attractive physical interpretation can be given to the present three-dimensional calculations as an extension of Born type calculations (sce BORN and HUANG, 1954: WEINER, 1983) which give the first order deformation gradient continuum elastic (local) description of monatomic perfect crystals. By including the second order gradient term in the continuum (non-local) description of the lattice, one can assess the possibility of existence as well as the stability of localized strain solutions. These localized deformation solutions occur at deformation gradients that are large enough to cause loss of ellipticity in the first order gradient model.

As an application to the general method presented, two different microstructures were examined. The first was designed so as to ensure ellipticity of the second order gradient model for the deformations of interest. The second model was motivated by problems in monatomic perfect crystals under plane strain. It was found that the inclusion of the second order gradient terms in the latter model does not restore ellipticity at deformation gradients where the first order gradient model loses ellipticity. Consequently, the higher order gradient model which accounts for the size of the microstructure - the lattice distance appears explicitly in the energy terms that include the contribution of the higher order deformation gradient--does not allow the existence of smooth localized strain solutions. This result should not come as a surprise. It is known that the localized deformation zone thickness in metallic crystals does not scale according to the lattice size, which is the only characteristic distance available in the present model. Based on some preliminary investigations, we believe that more accurate three-dimensional calculations for realistic lattices will also give the same conclusion, given that the size of the localized deformation zone in metals is orders of magnitude higher than the interatomic distance. This suggests that a different microstructural scale has to be considered in the derivation of a continuum model that accounts for the post-localization hehavior of the monatomic crystal.

At this point some additional comments about the calculation of higher order gradicnt continuum models for three-dimensional crystal geometries using realistic 
potentials are in order. It has long been known, going all the way back to the first atomistic models for elasticity, that the elastic moduli based on pair potentials with central forces between atoms often led to unrealistic conclusions. The firsi such result was the celebrated theoretical Poisson ratio prediction $v=1 / 4$ by Cauchy. The trouble goes even further, as seen in the calculations by MrLstern and Hill (1978) who show that for realistic Morse type pair potentials in f.c.c. or b.c.c. crystals one deduces negative shear moduli. This unacceptable result can be corrected by using some rather unrealistic long range potentials. A more attractive alternative is the use of three or many body interaction potentials. This approach has been successfully applied in the physics literature to calculate the elastic constants in monatomic perfect metallic crystals which match closely experimental observations [see for example Cousins (1973) for cubic crystals or IGARASH et al. (1991) for hexagonal close-packed metals]. Our general methodology proposed in Section 2.2 can be easily extended to include many body potentials instead of the two body ones for the interatomic force calculations in the discrete lattice models. Some preliminary 3-D calculations for b.c.c. crystals using Morse type pair potentials as well as three body potentials are under way with none of the results so far showing the possibility of localization at strains corresponding to the first loss of ellipticity in the first order gradient model.

The present work is part of a number of recent studies on the relations between the microscopic failure mechanisms and their corresponding macroscopic manifestations in solids with microstructure. In the interest of simplicity as well as mathematical consistency-disordered microstructures present substantial mathematical difficulties once non-linear phenomena are modeled and require a number of intuitive assumptions which are often difficult to prove--- efforts are focused on periodic microstructures and mechanical failure modes during a quasistatic loading process in the absence of rate, inertial or thermal effects. One part of these studies aims at relating macro- and microinstability mechanisms at the onset of failure (see ABEYARATNE and TRIANTAFYLLIDIS, 1984 ; TRIANTAFYLLIDIS and MAKER, 1985 ; GEYMONANT et al., 1993) where the failure mechanism at the micro-level is bifurcation buckling and the corresponding failure at the macro-level is shear band localization. Another part focuses on the post-failure range, where an attempt is made to find continuum theories that are capable of describing the solid's behavior after the onset of the initial instability. Results from the one-dimensional model by TRIANTAFYLLidis and BARDENHAGEN (1993) were encouraging and led to the present work in two and three dimensions. They provided valuable insight on what type of continuum behavior one has to expect in the postlocalization range in media with known microstructures. Similar problems arise in other engineering applications where the microstructure is no longer discrete. An investigation to extend the present methodology for deriving higher order gradient homogenized models from continua with periodic microstructures is currently under way.

\section{ACKNOWLEDGLMLNIS}

This work was partially funded by ALCOA. The authors are grateful to Dr Owen Richmond for stimulating discussions and his constant encouragement. 


\section{REFERENCES}

Abeyaratne, R. and Triantafyllidis, N. (1984) An investigation of localization in a porous elastic material using homogenization theory. J. Appl. Mech. 51, 481-486.

Aifantis, E. C. (1984) On the microstructural origin of certain inelastic models. $J$. Engng Mater. Technol. 106, 326-330.

Aifantis, E. C. (1987) The physics of plastic deformation. Int. J. Plasticity 3, 211-247.

Aifantis, E. C. and Serrin, J. B. (1983a) The mechanical theory of fluid interfaces and Maxwell's rule. J. Coll. Interf. Sci. 96, 517-529.

Alfanils, E. C. and Strkin, J. B. (1983b) Equilibrium solutions in the mechanical theory of fluid microstructures. J. Coll. Interf. Sci. 96, 530-547.

ASKar, A. (1985) Lattice Dynamical Foundations of Continuum Theories. World Scientific, Singapore.

BRAND, L. (1947) Vector and Tensor Analysis. John Wiley \& Sons, New York.

Born, M. and Huang, K. (1954) Dynamical Theory of Crystal Lattices (Chap. 3). Oxford University Press, Oxford.

Clifton, R. J. (1990) High strain rate of behavior of metals. Appl. Mech. Rev. 43, S9-S22.

Coleman, B. D. (1983) Necking and drawing in polymeric fibers under tension. Arch. Rat. Mech. Anal. 83, 115-137.

Coleman, B. D. and Hodgdon, M. L. (1985) On shear bands in ductile materials. Arch. Rat. Mech. Anal. 90, 219-247.

Cousins, C. S. G. (1973) Evidence for a short range three body interaction in copper. J. Phys. F. Metal. Phys. 3, 1915-1920.

Eringen, A. C. and Suhubi, E. S. (1964) Nonlinear theory of simple micro-elastic solids-I. Int. J. Engng Sci. 2, 189-203.

Gantmacher, F. R. (1974) The Theory of Matrices, II (Chap. 15). Chelsea, New York.

Geymonant, G., Müller, S. and Triantafyllidis, N. (1993) Homogenization of nonlinearly elastic materials, microscopic bifurcation and macroscopic loss of rank-one convexity. To appear in Arch. Rat. Mech. Analysis.

Hadamard, J. (1903) Lecons sur la Propagation des Ondes et les Equations de l'Hydrodynamique (Chap. 6). Hermann, Paris.

HiLl, R. (1962) Acceleration waves in solids. J. Mech. Phys. Solids 10, 1-16.

Igarashi, M., Khantha, M. and Vitek, V. (1991) $\mathrm{N}$-body interatomic potentials for hexagonal close-packed matals. Phil. Mag. B 63,603-627.

K NOWles, J. K. and SternberG, E. (1977) On the failure of ellipticity of the equations for finite elastostatics plane strain. Arch. Rat. Mech. Anal. 63, 321-336.

Kunin, I. A. (1982) Elastic Media with Microstructure-I (One-Dimensional Models). Springer, Berlin.

Leroy, Y. M. and Molinari, $\Lambda$. (1993) Spatial patterns and size effects in shear zones: a hyperelastic model with higher-order gradients. J. Mech. Phys. Solids 41, 631-664.

Mandel, J. (1966) Conditions de stabilité et postulat de drucker. In Rheology and Soil Merhanics (ed. I. KravtrhFnko and P. M Sirifys), pp 58-68. Springer, Berlin.

MARCINIAK, Z and KUCZYNSKI, K. (1967) Limit strains in the process of stretch forming sheet metal. Int. J. Mech. Sci. 9, 609-625.

Milstein, F. and Hill, R. (1977) Theoretical properties of cubic crystals at arbitrary pressure-I. Density and bulk modulus. J. Mech. Phis. Solids 25, 457-477.

Milstein, F. and Hill, R. (1978) Theoretical properties of cubic crystals at arbitrary pressureII. Shear moduli. J. Mech. Phy's. Solids 26, 213-239.

Milstrin, F. and HiLl, R. (1979) Theoretical properties of cubic crystals at arbitrary pressure-III. Stability. J. Mech. Phy's. Solids 27, 255-279.

Mindlin, R. D. (1964) Micro-structure in linear elasticity. Arch. Rat. Mech. Anal. 16, 51-78.

Mindolin, R. D. (1965) Second gradient of strain and surface-tension in linear elasticity. Int. J. Solids Struct. 1, 417-438.

Molinari, A. and Clifton, R. J. (1987) Analytical characterization of shear localization in thermoviscoplastic solids. J. Appl. Mech. 54, 806-812. 
Ric 1, J. R. (1976) The localization of plastic deformation. In Theoretical and Applied Mechanics. Conference Procedings I.U.T.A.M. (ed. W. T. Korme). pp. 207-220. North-Holland. Amsterdam.

Stiltibi, E. S. and Erincilin. A. C. (1964) Nonlinear theory of micro-elastic solids II. Int. I. Engny Sci. 2, 389404.

Thomas. T. Y. (1961) Plastic Flow and Fracture in Solids. Academic Press. New York.

Thompsom, J. M. T. and SHorrock, P. A. (1975) Bifurcational instability of an atomic lattice. J. Mech. Phis. Solids 23, 2137.

Tol:pin. R. A. and Gazis, D. C. (1965) Surface effects and initial stress in continuum and latice models of elastic crystats. In Proc. Int. Conf. on Lathice Dynamics, Copenthagen. Augus1, 1963 (ed. R. F. Whlllis), pp. 597-605. Pergamon Press. Oxford.

Triantafyleidis, N. and Allavis, E. C. (1986) A gradient approach to localization of deformation. I Hyperelastic materials. I. Elesticity 16, 225 237.

Triantafyllidis, N. and Bardenhagen, S. (1993) On higher order gradient continuum theories in $1-\mathrm{D}$ nonlinear elasticity. Derivation from and comparison to the corresponding diserete models. To appear in J. Elasticity.

Triantafyllidis. N. and Makek. B. N. (1985) On the comparison between mieroscopic and macroscopic instability mechanisms in a class of fiber-reinforced composites. $J$. Appl. Mech. $52,794.800$.

TVtrgando, V., Nlidiliman, A. and Lo, K. K. (1981) Flow localization in the plane strain tensile test. J. Medh. Phis. Solids 29, 115 142.

VAN dER WAal.S, J. D. (1893) The thermodynamic theory of capillarity under the hypothesis of a continuous variation of density (in Dutch). Verhandel. Konink. Aked. Werten. Amsterdem (sec. 1) 1 .

Whing. J. H. (1983) Statistical Mechamics of Elasticit! (Chap. 4). John Wiley \& Sons. New York.

\section{APPENDIX}

The Cauchy index $I_{u}^{h}[R(x)]$ of a real rational function $R(x)$ between limits $a$ and $b$ is the difference between the number of jumps from $-x+10+\infty$ and that of jumps from $+x$ to $-x$ as the argument changes continuously from $a$ to $b$. In particular, for polynomial $p_{n}(x)=A\left(x-\alpha_{1}\right)^{n_{1}} \ldots\left(x-\alpha_{4}\right)^{n_{4}}, n=n_{1}+\cdots+n_{q}$, with $q$ distinct roots $\alpha_{1}, \ldots, \alpha_{q}$, the first $l$ of which are real:

$$
\begin{aligned}
& p_{\prime \prime}^{\prime}(x) \\
& p_{n}(x)
\end{aligned}=\sum_{i=1}^{\prime \prime} n_{i} n_{i}=\sum_{i}^{1} n_{i} n_{i}+\tilde{R}(x)
$$

where $\hat{R}(x)$ is a real rational function without real poles. It is evident then that $l$, the number of distinct real roots of $p_{n}(x)$ is equal to $I^{1} ;\left[p_{\prime \prime}^{\prime}(x) p_{n}(x)\right]$.

The Cauchy index $I^{+}:\left[p_{\prime \prime}^{\prime}(x) p_{n}(x)\right]$ may be computed by constructing a Stum chain, which is a sequenec of polynomials $f_{m \prime \prime}(x), f_{m},(x) \ldots, f_{0}(x)$ satisfying:

(1) $f_{i},(x) f_{i},(x)<0$ when $f,(x)=0$. i.c. $f_{i}, f(x)$ and $f_{f},(x)$ are different from zero and of opposite sign when $f(x)$ passes through zero:

(2) $f_{1},(x)=C . C \neq 0$. i.c. $f_{10}(x)$ is different from zero on $(-x, x)$.

If $l(x)$ denotes the number of changes in sign at $x$ of the sequence $f_{m}(x), f_{m,},(x), \ldots, f_{0}(x)$, then as $x$ is varied from $-x 10+x V(x)$ is not affected by polynonials $f(x)$ passing through zero for $j<m$ by (1). As $f_{m}(x)$ passes through zero one variation in sign is lost or gained as the ratio $f_{m},(x) f_{m}(x)$ goes from $-x$ to $+x 10-x$ respectively. Hence the classical theorem of Sturm. applied to the entire real axis. states 


$$
I^{+}:\left[\frac{f_{m-1}(x)}{f_{m}(x)}\right]=V(-x)-V(+x) \text {. }
$$

The number of distinct roots of $p_{n}(x)$ may then be found by constructing a Sturm chain using $f_{n}(x)=p_{n}(x), f_{n-1}(x)=p_{n}^{\prime}(x)$, and applying the theorem of Sturm. Such a sequence of polynomials may be constructed as follows. Let $q_{i}(x)$ and $-f_{-2}(x)$ be the quotient and remainder respectively, obtained by dividing $f_{i}(x)$ by $f_{j},(x)$. Then the sequence of polynomials:

$$
\begin{aligned}
f_{n}(x)= & q_{n}(x) f_{n},(x)-f_{n, 2}(x), \\
f_{n 1}(x)= & q_{n-1}(x) f_{n}(x)-f_{n, 3}(x), \\
& \vdots \\
f_{2}= & q_{2} f_{1}-f_{0} . \\
f_{1}= & q_{1} f_{11}, \\
f_{0}= & C
\end{aligned}
$$

is a Sturm chain if $C \neq 0$. If $C=0$ then the first $f(x) \neq 0$ is a common factor in the sequence, and $f_{n}(x) / f_{j}(x), f_{n},(x) / f_{j}(x), \ldots, f_{i+1}(x) / f_{i}(x), 1$ is a Sturm chain with the same number of distinct roots, $l$, where

$$
l=V(-\infty)-V(+\infty)
$$

